\title{
Drag reduction by additives in curved pipes for single phase liquid and two phase flows: A review
}

\author{
Paul Ayegba ${ }^{1}$, LAWRENCE EDOMWONYI-OTU², Nurudeen Yusuf ${ }^{3}$, and Abdulkareem \\ Abubakar ${ }^{4}$ \\ ${ }^{1}$ University of California Berkeley \\ ${ }^{2}$ Delta State University - Oleh Campus \\ ${ }^{3}$ Bayero University Faculty of Technology \\ ${ }^{4}$ Ahmadu Bello University Faculty of Engineering
}

May 5, 2020

\begin{abstract}
A review of investigations on the effect of drag-reducing agents in curved pipe flows is presented in this work. Proposed mechanisms of drag reduction, as well as factors that influence their effectiveness also received attention. In addition, this review outlined proposed friction factor and fluid flux models for flow of drag-reducing agents in curved pipes. It was shown in this report that significant drag reduction in curved pipes can be achieved using drag-reducing agents. Drag reduction by additives in curved pipes are generally lower than the corresponding drag reduction in straight pipes. It decreases with increase in curvature ratio and is more pronounced in the transition and turbulent flow regimes. Drag reduction depends strongly on the concentration of polymers and surfactants as well as the bubble fraction of micro-bubbles. It is also reported that drag reduction in curved pipes depends on other factors such as temperature and presence of dissolved salts. Maximum drag reduction asymptote differed between straight and curved pipes and between polymer and surfactant. Due to the limited studies in the area of drag reduction for gas-liquid flow in curved pipes no definite conclusion could be drawn on the effect of drag-reducing agents on such flows. A number of questions remain such as the mechanism of drag reduction in curved pipes and how drag-reducing agents interact with secondary flows. Hence, some research gaps have been identified with recommendations for areas of future researches.
\end{abstract}

\subsection{Introduction}

Locations of petroleum wells are sometimes several kilometres from processing plants. There is therefore need to transport single and multi-phase fluids through pipes, including pipe fittings such as bends, to processing plants and for separation [1]-[3]. A large percentage of the energy cost in petroleum production and transport results from pressure losses. Most of the pressure losses in pipeline flows are associated with the production of turbulence eddies resulting in non-axial components of flow. Unlike laminar flows where pumping power is directed at providing axial unidirectional fluid flow, turbulent flows are characterised by both axial and radial flows. The implication of this is loss of pumping power or increased drag. A common view is that any process mechanism that results in flow laminarization would also result in drag reduction [4].

Drag reduction (DR) is a process of reducing pressure losses associated with flows [5]. Additives, called drag-reducing agents (DRAs), are often used for drag reduction. After the pioneering work credited to Tom 
[5], several studies have examined the effect of DRAs on liquid flows through straight pipes and channels of various orientations [7], [8]. A few others investigated this effect in curved pipes [9]-[11]. Other methods of drag reduction involving pipe modifications such as riblets, dimples, wavering walls and amenable surfaces are also common [12]-[14].

Drag reduction (DR) as originally defined by [15] is given by Eq. (1).

$\operatorname{DR}(\%)=\frac{\left(\frac{d p}{d 1}\right)_{s}-\left(\frac{d p}{d 1}\right)_{\text {DRA }}}{\left(\frac{d p}{d 1}\right)_{s}} \times 100 \%(1)$

where $\left(\frac{\mathrm{dp}}{\mathrm{dl}}\right)_{s}$ and $\left(\frac{\mathrm{dp}}{\mathrm{dl}}\right)_{\text {DRA }}$ are frictional pressure gradients for solvents and DRA solution respectively, under the same flow conditions. Where the viscosity and density of solvent and polymer solution are almost the same, Eq. (2) gives an equivalent measure of drag reduction.

$\operatorname{DR}(\%)=\frac{f_{s}-f_{\text {DRA }}}{f_{s}} \times 100 \%(2)$

where $f_{s}$ is the fanning friction factor before the addition of DRA. $f_{D R A}$ is the fanning friction factor after the addition of DRA.

$f=\frac{\tau_{w}}{\frac{1}{2} \rho U^{2}}(3)$

The wall shear stress $\tau_{w}$ is given by

$\tau_{w}=\frac{d p}{4 l}(4)$

where $d$ is the internal diameter of pipe and $P$ is the frictional pressure drop over the pipe length $l$.

Eqs. (1) and (2) are referred to as pressure drop drag reduction and friction factor drag reduction [16].

A measure of drag reduction, in curved and straight pipes, called turbulence reduction drag (TRD) given by Eq. (5) is sometimes used [17].

$\operatorname{TRD}(\%)=\frac{f_{T}-f_{T_{-} D R A}}{f_{T}-f_{L}} \times 100 \%(5)$

where $T$ and $L$ denote turbulent and laminar flow of the solvent respectively.

The definition given by Eq. (5) enables comparison of only the degree of turbulence suppression in curved and straight pipes. In general, the difference between Eq. (2) and (5), for straight pipes is small. However, the respective difference is large in the case of flow in curved pipes due to suppressed turbulence and secondary flow effects [18]. It should be stated that at the same Reynolds number of flow, the degree of turbulence in straight pipes is higher than that in curved pipes [19].

Drag-reducing agents also influence turbulent heat transfer [20]-[22]. In certain applications, the effect of DRAs on heat transfer reduction (HTR) outweighs its effect on drag reduction [23]. Besides heat transfer and drag reduction, DRAs affects flow structure, phase-distribution and flow regime transitions [24]-[27].

Till date, most of the drag reduction studies have focussed on flows through vertical, horizontal, inclined and undulated pipes. Application of DRAs for flows in curved pipes has received little attention. Moreover, the flow of single and multiphase fluids through curved pipes is a common occurrence in the petroleum and chemical industries. Such a flow is associated with large pressure drop and pressure fluctuations among other effects [10]. It is important to gain insight into drag reduction in curved pipes to improve the economics of pipeline design and operation. Fsadni [27] provided a brief review of pressure drop reduction studies for flow in helical coils. Besides this review, the Authors are not aware of any other reviews pertaining to drag reduction in curved pipes. Hence this work is devoted to the review of existing research on single and two-phase drag reduction for flows through curved pipes. 


\subsection{Drag reduction in curved pipes}

Virk [28] published an extensive review on drag reduction in straight pipes. The paper highlighted some important aspects of drag reduction such as mechanism of polymer drag reduction, turbulence structure and velocity profile. The work also pioneered the concepts of maximum drag reduction (MDR) and drag reduction envelop. The Virk's envelop for polymer drag reduction in straight pipes is shown in Fig. 1 on the Prandtl-Karman coordinates. Eqs. (6) - (8) give the equations for laminar flow, turbulent flow and maximum drag reduction asymptote. The maximum drag reduction law holds irrespective of polymer specie used, its concentration or molecular weight [29].

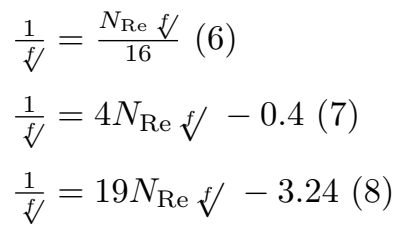

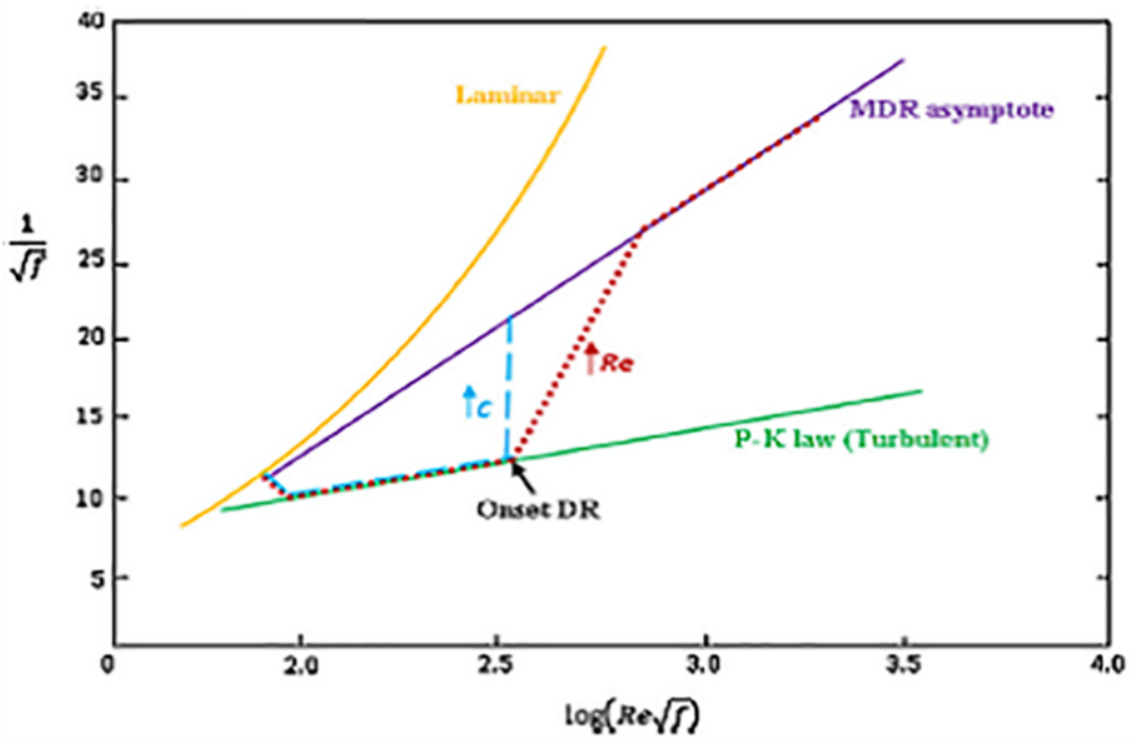

Figure 1. Virk's envelop in Prandtl-Karman coordinates for drag reduction in straight pipes Source:[30]. 


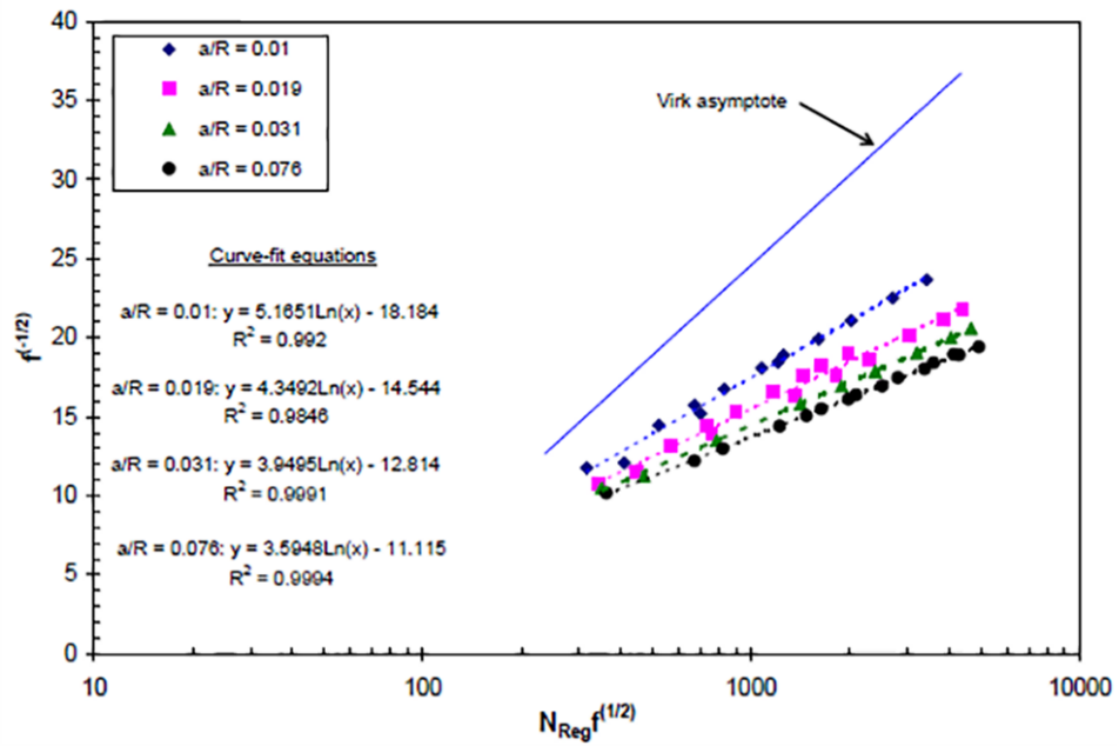

Figure 2. MDRAs for various curvatures of coiled pipe plotted on the Prandtl-Karman coordinates. Source: [31].
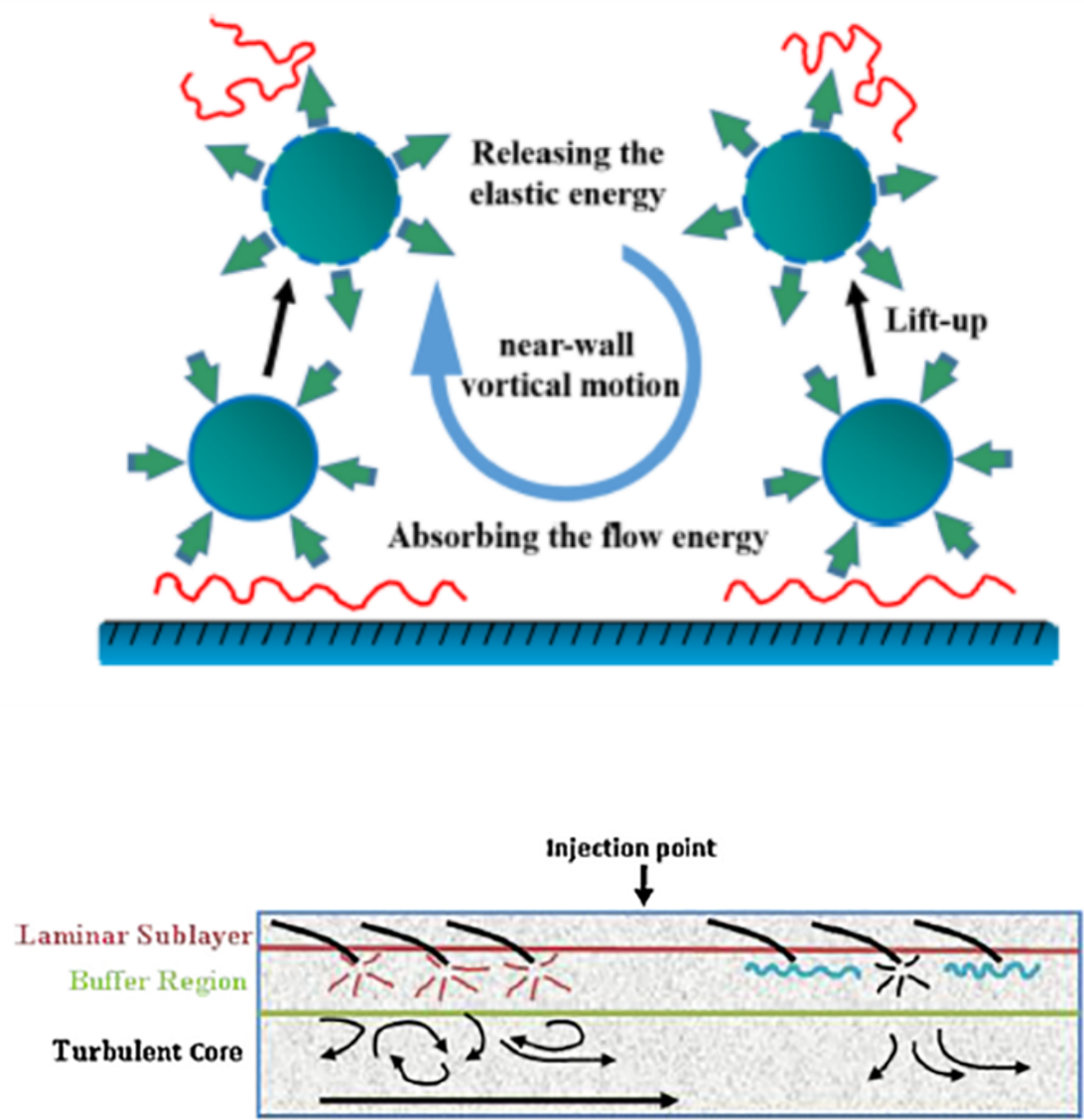
$\mathrm{a} \mathrm{b}$

Figure 3. (a) Schematic representation of polymer-induced turbulent drag reduction (DR) mechanism [32] (b) Illustration of turbulence suppression mechanism Source: [13].

Between the Prandtl-Karman Law and the maximum drag reduction curve is a roughly linear polymeric regime characterised by the wall shear $\operatorname{stress}\left(\tau_{w}\right)$ and the increment in slope $(\delta)[33]$. This regime is represented by Eq. (9).

$$
\frac{1}{f}=(4.0+\delta) \operatorname{Re} f /-0.4-\delta\left[\frac{2 d}{v_{s}}\left(\frac{\tau_{w}^{*}}{\rho}\right)^{\frac{1}{2}}\right]
$$

The centrifugal forces associated with flow in curved pipes results in secondary flows which appear in the form of vortices [34]. Centrifugal force causes faster-moving fluids in the middle of the pipe to move to the outer wall while fluids in the outer wall to move to the centre resulting in secondary flow [10]. These vortices flow behaviour results in flow fluctuations and higher pressure drop in curved pipes compared with that in straight pipes of equivalent length. The higher pressure drops observed in curved pipes prompted Shah and Zhou [30] to propose a modified Virk's envelop. They replaced the Prandtl-Karman Law (Newtonian line in Fig. 1) with a Newtonian friction factor correlation for coiled tubing given by Srinivasan [32]. Findings have revealed that maximum drag reduction asymptote (MDRA) for curved pipes is lower than that of straight pipes and depends on the curvature ratio of the pipe. Shah and Zhou [33] proposed an expression for MDRA for flow of drag-reducing polymers (DRPs) in curved pipes as a function of curvature ratio given by Eq. (10). Fig. 2 shows the MDRAs for coils of various curvatures, as determined by Eq. (10).

$\frac{1}{f}=A N_{\operatorname{Re}} \sqrt[f]{ }+B(10)$

where; $A=\left[c_{1}+c_{2}\left(\frac{a}{R}\right)^{0.5}\right]^{-1}, \quad c_{1}=0.053109965, c_{2}=0.29465004$ and

$B=\left[c_{3}+c_{4}\left(\frac{a}{R}\right)^{0.5}\right]^{-1}, \quad c_{3}=0.0309447, c_{4}=0.245746$

when $\left(\frac{a}{R}=0\right), \quad A=18.83$ and $B=32.32$, and Eq. (10) reduces approximately to the Virk's MDRA.

Based on the redefined MDRA for curved pipes, Shah and Zhou [33] described a new drag reduction envelop. This drag reduction envelop is bounded by three lines - the laminar flow line, the MDRA for curved pipe and the zero-drag reduction line given by the Srinivasan [32] correlation for Newtonian turbulent flow in curved pipes. The laminar flow correlation chosen for their work was that of Liu and Masliyah [34].

Studies have shown that phenomenological models for MDRA, developed for polymers, are not applicable to surfactants. An interesting characteristic of surfactants is their higher shear viscosity compared to polymer solutions. This makes surfactant solution more shear rate dependent and makes the definition of the Reynolds number all the more difficult [21]. Zakin et al. [35] showed that fanning friction factor curves of most surfactants in straight pipes lies below the Virk MDRA. They proposed an MDRA for surfactant solutions in straight pipes given by:

$f=0.32 N_{\operatorname{Re}}^{-0.55}(11)$

Their work did not account for how viscosity depends on the shear rate in surfactants. Aguilar et al. (2006) used surfactant with viscosity similar to that of the solvent and recorded friction factors slightly lower than those given by the Zakin MDRA. They proposed a new correlation for MDRA given by;

$f=0.18 N_{\operatorname{Re}}^{-0.50}(12)$

Surfactant solutions exhibit higher MDRA than polymers. Kamel and Shah [36] therefore extended Zakin et al. [35] MDRA for straight pipes to curved pipes and proposed a correlation for MDRA for surfactant in coiled pipes given by Eq. (13). 
$f=\left[-32200.42\left(\frac{a}{R}\right)^{3}+1830.62\left(\frac{a}{R}\right)^{2}+0.32\right] N_{R e^{\prime}}^{\left[7210.95\left(\frac{a}{R}\right)^{3}-316.97\left(\frac{a}{R}\right)-0.55\right]}$

They further suggested a modified maximum drag reduction envelop for surfactant in coiled pipes bounded by Liu and Masliyah [34]'s equation for laminar flow, the Srinivasan et al. [32] correlation and Eq. (13).

\subsection{Drag-reducing Agents (DRAs)}

Drag-reducing agents include additives such as polymers, surfactant, fibres and micro-bubbles. The use of polymer as a drag-reducing agent is most common because only small concentrations is needed to produce drag reduction [13], [39], [40]. Drag-reducing agents can either be soluble or insoluble resulting in homogeneous and heterogeneous fluids mixtures respectively [41]. The benefits of DRAs include reduced operation cost and ease in application [42]. Its application in oil and gas ranges from petroleum product transport to enhance oil recovery [43].

\subsubsection{Polymer DRAs}

Synthetic and natural polymers are classes of polymer DRAs. Examples of synthetic polymers include; polyethylene oxide (PEO), polyisobutylene (PIB), polyacrylamide (PAM), partially hydrolysed polyacrylamide (HPAM) etc. Synthetic polymers generally produce high percentage drag reduction. They are, however, mostly non-biodegradable thereby posing environmental challenges. Natural polymers include; carboxymethylcellulose (CMC), guar gum (GG), xanthan gum (XG), tragacanth, karaya, locust bean, chitosan and okra [14]. Natural polymers are biodegradable thus making them environmentally friendly [41]. However, this biodegradability reduces their shelf life thus reduces their effectiveness for long-distance transport. Grafting the artificial polymers into the rigid structures of natural polymers have been suggested as a means of controlling biodegradation [14], [44]. Recent advances in polymer technology have seen the rise in high performance biodegradable polymers. Some of the recent synthesis have been centred around improved cross-linking of polymer chains [45], [46]. A common characteristic of DRAs is the increase in efficiency with increase in molecular weight of polymer. A drawback of polymers DRAs is their susceptibility to both chemical and mechanical degradation. High molecular weight $\left(\mathrm{M}_{\mathrm{wt}}>10^{6}\right)$ polymers are the most commonly employed DRAs possibly because of their unique rheological properties which makes them effective and economical [14]. Various theories exist seeking to explain the mechanism of polymer drag reduction. These theories includes those based on shear thinning, viscoelasticity, vortex stretching, molecular stretching, flow anisotropy and turbulence suppression [16], [32].

A number of researchers have tried to explain the mechanism of polymer DR by molecular stretching of polymer molecules. In this model, the shear-hardening characteristic of drag-reducing polymers (DRPs) is assumed to increase resistance to extensional flow, thereby inhibiting turbulent burst at the near wall region. The Lumley [44] model, which is based polymeric chain extension, suggest that DR involves increased elongational viscosity. This results in increased thickness of the viscous sub-layer which dampens and suppresses small eddies and turbulent fluctuations. The overall effect is higher turbulence dissipation, reduction of both velocity gradient and shear stress near the wall and consequently reduction of drag. It has also been suggested that stretching of polymer molecules results in the storage of elastic energy (see Fig. 3a) emanating from flow very close to the wall [48]. Thus if there is sufficient relaxation time, the elastic energy is transported to the buffer layer and dissipated there by the vortex motion resulting in DR ([49].

A number of proposed DRP drag reduction mechanisms are based on polymer's spring like behaviour. A bead-spring model was used by Armstrong and Jhon [47] to describe the mechanism of DR. The polymer molecule is assumed to be a chain of identical beads linked by an arbitrary spring potential. Here the effect of the stochastic velocity field on the polymer molecule is associated with arenormalisation of the connector potential and the dumb-bell probability density is derived for the arbitrary connector potential. At certain degree of turbulence, the second moment of the probability density becomes infinite. The renormalisation

of the connection potential between the beads reduces the connection force, thus making the beads extend (or polymer molecules expand). A mechanism analogues to the dumb-bell model wherein stretched polymer 
molecule are simplified as springs with masses at their ends was also proposed by [49]. The theory assumes that there is a balance between centrifugal stretching force and centripetal restoring force acting on rotating polymer chains. The rotational flow kinetic energy is converted to polymer elastic energy and subsequently becomes damped by the surrounding viscous fluids when the polymer relaxes.

A common view is that interaction of polymer with turbulence (resulting in flow laminarization) is the main reason for its efficiency as a drag-reducing agent [42]. The complex rheological properties of DRPs such as viscosity and elasticity play important role in the process [14]. The non-axial component of turbulent flows results in wasteful turbulent eddy dissipation and the implication of this is increased drag [16]. The ability of DRP to induce flow laminarizationtranslates to reduction of wasteful energy dissipation and consequently DR. In effect, the action of DRPs in flow laminarization is to reduce radial velocity fluctuations and Reynolds stresses [32], [41], [42].

The anisotropic behaviour of DRP solutions, where shear rate, structure and viscosity of the solution are directionally dependent, have been used to explain polymer DR. Here the effect of DRPs is to alter the turbulence structure and reduce drag [51]. Models based on the finite elastic non-linear extensibility-Peterlin (FENE-P) have also been used to explain the mechanism of polymer DR. Here pre-averaging approximation is applied to a suspension of non-interacting finitely extensive non-linear elastic dumb-bells, thus accounting for the finite extensibility of the molecule [52]. The FENE-P model has been used by Li et al. [50] as viscoelastic polymer conformation tensor equation.

A few numerical simulation studies have been carried out to shed more light on DR mechanism. In the Brownian dynamic simulation studies of Terrapon et al. [51] it was demonstrated that polymers experience significant straining around the vortices resulting in molecular stretching. As polymer molecules stretches around the vortices, by upward and downward fluid motion, there is extraction of energy from the near-wall vortices. Numerical studies has also been carried out to describe the systematic storage and release of energy to the flow by polymer [55], [56]. Energy storage occurs at the near-wall vortices, while the release of energy occurs at the very-near-wall region. Numerical studies was also used to show that polymer mixing acts as a relaxation mechanism for DR [57]. Direct numerical simulation was used to investigate the roles of shear stress/shear rate anisotropy and elasticity on DR [58]. The hypothesis is that, when polymer stretches, the viscous anisotropic effect produces change in turbulent structures and change in entropy which in turn results in DR. To shed more light on the mechanism of DR and explain certain observed behaviours, various studies have been carried out using laser Doppler velocimetry (LDV) and particle image velocimetry (PIV) [41], [59]-[62].

Overall, it appears that more than one of the suggested mechanisms is involved in DR. Notwithstanding the mechanism(s), polymers do stretch in the flow thereby absorbing the energy in the streak. This inhibits turbulent burst formation (Fig. 3b) in the buffer region and results in turbulence suppression.

The above reports details efforts to explain the DR mechanism via investigations of flows in straight pipes. Similar to straight pipes, DR by polymer solutions in curved pipes and channels have been linked with the dampening of turbulent intensities [63]. A few suggested mechanisms for polymer drag reduction in the laminar flow regime of curved flow exist. The general understanding is that for DRAs to be effective in the laminar flow regime of curved pipe flows, there must be an interaction between the DRAs and secondary flow stream lines. A few early studies investigated the effect of DRAs on secondary flows but the conclusions are inconsistent and mostly speculative [64]-[66]. Frictional losses as well as secondary flow losses contributes to pressure losses in hydrodynamically developed flows in coils. In the case of undeveloped flows in and after bends, additional form-drag exist due to flow redistribution. The effect of DRAs on each of these competing forces is a subject of investigation by the authors using a dedicated flow loop at the University of California Berkeley. 


\subsubsection{Surfactant DRAs}

Surfactants are surface-active chemical agents of relatively low molecular weight which alters the surface tension of the liquid in which it dissolves [67]. They assume various structures in solution such as spherical micelles, rod-like micelles, crystals, emulsions and vesicles depending on the concentration, temperature, salinity etc. [38]. The classes of surfactants are ionic (examples; anionic, cationic and zwitterionic) and non-ionic surfactants. When compared to polymer they have higher resistance to mechanical degradation [68] and are thermodynamically stable [23]. This is due the their ability to self-repair after degradation [21], [69]. The efficiency of surfactants in reducing drag depends on its concentration, temperature, geometry of flow channel, size of micelles and bond strength. Some early investors [70], [71] linked the mechanism of drag reduction in surfactants to the viscoelastic rheology of the solution. However, drag reduction has since been observed in non-viscoelastic surfactants [72]. The ability of surfactants to act as drag reducers is associated with the formation of thread-like micelles. These micelles changes the structure of turbulent flow at the near wall region [34], [68]. It has been suggested that surfactants drag reduction is achieved when micelles, under shear stress, line up in the direction of flow and build a huge network structure (the so-called shear-induced state) [42], [73]. This leads to a damping of radial turbulence and subsequently reducing pressure loss. Fig. 4a shows surfactant molecules and micelles structures while Fig. 4b show the transmission electron microscope (TEM) image of surfactant micelles. Different surfactants show different response or characteristics under the influence of shear. For example, the viscosity of Habon G decreased under prolonged shearing or mixing while that of the mixture Ethoquad $\mathrm{T}$ 13/ sodium salicylate (NaSal) increased after prolonged shearing in a rotational viscometer. The effective velocity range for which various surfactants produce drag reduction depends on the concentration and age of the surfactants [74]. The effectiveness of surfactants as drag-reducing agents is negatively influenced by disturbances in the flow, though sensitivity of surfactants to disturbances differs. This is important in bend-flow applications where there are high disturbances resulting from the bend. As reported by Gasljevic and Matthys [9], additional drag results from the flow of surfactant solutions in the region of high flow disturbance after the bend.

Cationic surfactants are by far the most commonly used drag-reducing surfactants $D R S$. Cationic surfactants combined with suitable counter-ions are effective drag reducers [75]. The applicability of anionic surfactants in aqueous or hydrocarbon solutions depends on their molecular weight. In general, low-molecular weight surfactants are used as drag-reducing agents. Very low-molecular weight $(<10$ carbon atoms in chain) anionic surfactants are too soluble to have substantial surface effect and thus results in small drag reduction [42]. The surface-active portion of zwitterionic surfactants carry opposing charges on it as well as a subgroup derived from imidazoline. Zwitterionic surfactants are more environmentally friendly than the cationic ones. However at the recommended (low) concentration, they are very sensitive to upstream disturbances (as is common in bends) in the flow which may impede their drag-reducing capabilities [74]. Non-ionic surfactants are known to be chemically, mechanically and thermally stable in comparison with ionic surfactants. In addition non-ionic surfactants do not precipitate in the presence of calcium ions [41]. Non-ionic surfactants are only applicable over a limited range of temperature and concentrations and may be susceptible to chemical degradation [14]. Glycolic acid ethoxylate, Arquad 16-50 Cetyltrimethylammonium chloride (CTAC), Ethoquad O12, Soya- $\mathrm{N}\left(\mathrm{CH}_{3}\right)_{3} \mathrm{Cl}$ and Sodium oleate are some examples of commonly used surfactants [14]. Van der Plas [73] recently defined some essential characteristics required by viscoelastic surfactants for them to be effective DRAs in petroleum applications. It is safe to assume that insight into micelle formation and rheological properties of surfactants are essential to understanding the mechanism for drag reduction of surfactant solutions. Due to the high shear stresses observed in curved pipes, surfactants are more suitable as dragreducing agents for flow through bends than polymers [53].

\subsubsection{Micro-bubbles DRAs}

The application of air in micro-bubbles drag reduction is environmentally friendly and cheaper compared to polymers and surfactants [77], [78]. Micro-bubbles have diameters less than ten-microns and exhibit behaviours different from those of larger size bubbles. These differences are seen in their chemical and physical characteristics such as the tendency to remain suspended in the liquid phase over longer periods 
of time [78]. The first work published on the application of micro-bubbles as drag-reducing agents was by [79]. The mechanism of drag reduction by micro-bubbles is not yet well understood. Similar to other drag reduction techniques, the purpose of micro-bubble injection is to alter the structure of the boundary layer. It had been suggested that micro-bubbles reduce drag by altering both laminar and turbulent boundarylayer characteristics [79]. It has been reported that, injecting air bubbles results in an increase in kinematic viscosity and decrease in the turbulent Reynolds number in the buffer layer [80]. This results in thickening of the viscous sub-layer and decrease in the velocity gradient at the wall. Hassan and Ortiz-Villafuerte [74] used particle image velocimetry (PIV) to study the effect of injecting low void fraction micro-bubbles into the boundary layer of a channel flow. Some of their results showed some similarities with drag reduction behaviour by polymers or surfactants as well as reports of some earlier investigations [80], [81]. These similarities include thickening of the buffer layer as well as upward shift of the log-law region. They stated that the micro-bubble layer formed at the top of the channel was not responsible for the drag reduction recorded. This micro-bubble layer served to reduce the slip between the micro-bubbles and the liquid. The major contribution to drag reduction is the accumulation of micro-bubbles in a critical zone within the buffer layer. The interaction of micro-bubbles with turbulence in the buffer layer is responsible for the observed DR. in general, injection of micro bubbles reduces turbulent energies with the shear in the boundary layer remaining unchanged [82]. There appears to be some agreement on the mechanism of micro-bubble drag reduction especially as it relates to thickening of the viscous sub-layer and turbulence suppression.

\subsection{Effect of polymer and surfactant DRAs on pressure drop and friction factor for single phase flows in curved pipes and channels}

Majority of reports suggest that the effectiveness of drag-reducing agents is higher in straight pipes than in curved pipes [9], [11], [23]. The reduced drag reduction in curved pipes, compared with straight pipe has been attributed to secondary flow resulting from the centrifugal forces [23]. The lower drag reduction in curved pipes is the result of the differences between the extended laminar and extended turbulent flow friction factor curves which are much larger for straight pipes.

\subsection{Effect of polymer and surfactant DRAs on pressure drop for single phase liquid flows in coiled pipes}

A number of interesting reports on the application of polymers and surfactants as drag-reducing agents for flow through coils exist in open literature. In general, the effectiveness of polymers and surfactants in reducing pressure loss is dependent on pipe geometry, flow rate as well as type, concentration and molecular weight of the drag-reducing agents. It also depends on temperature, presence of dissolved salts and phase distribution before and after adding the drag-reducing agent [76].

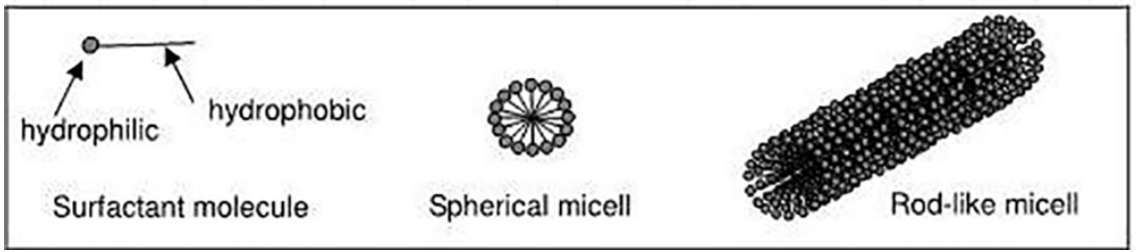

a 


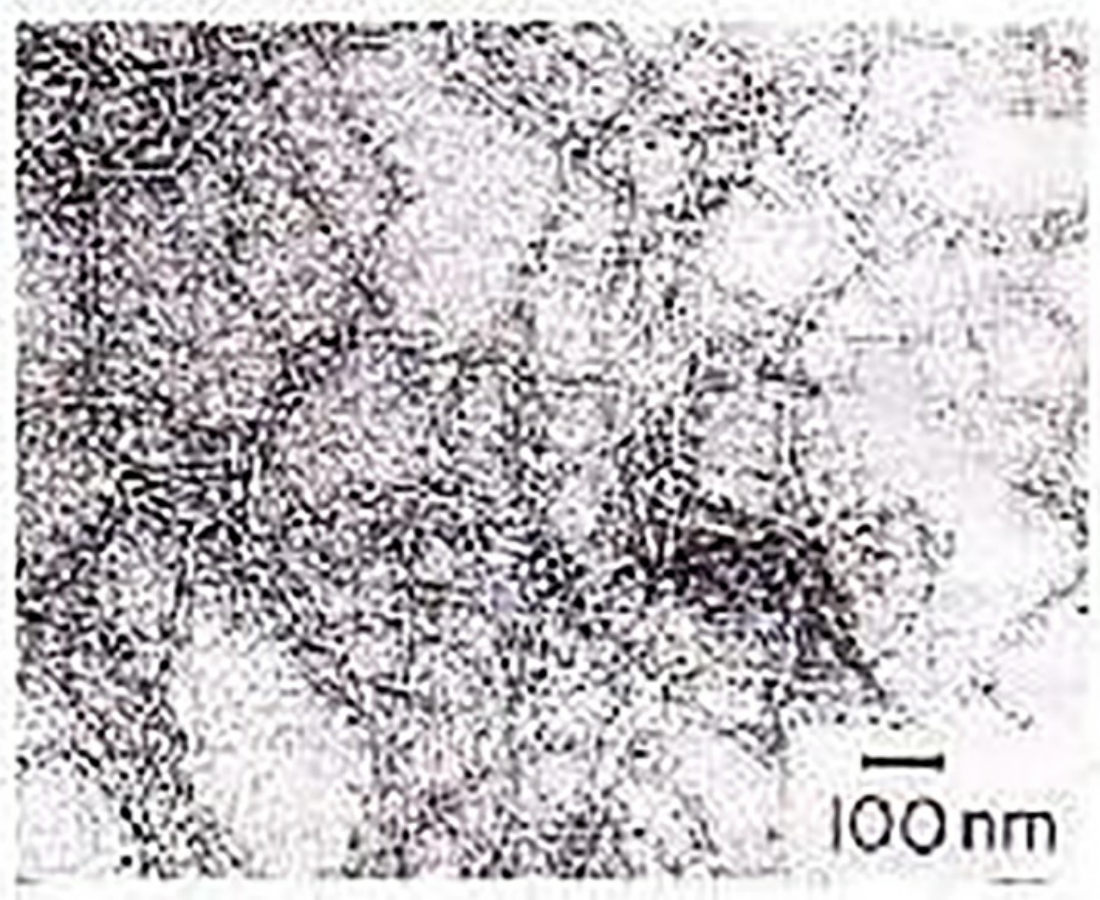

b

Figure 4. a. Surfactant molecule and micelle structures Source:[69] b. Rod like structures of surfactant micelles Source:[69].

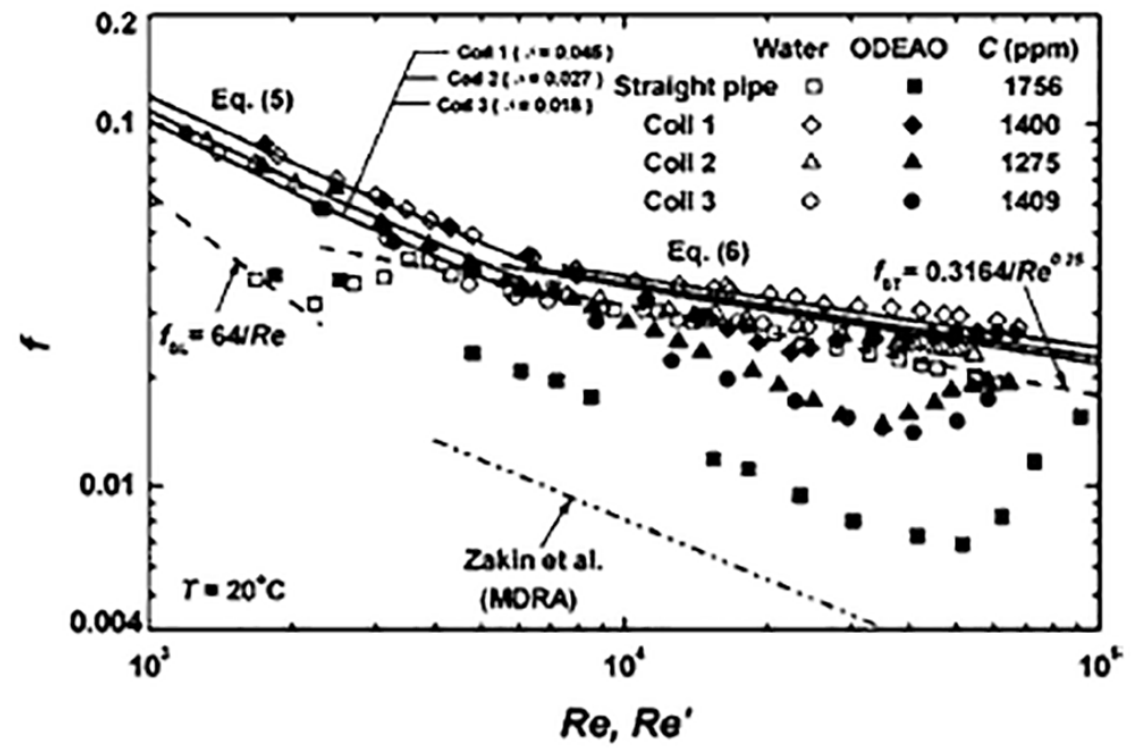

Figure 5. Friction factor versus Reynolds number for water and ODEAO surfactant solution in both straight and coiled pipes. Source:[23]. 


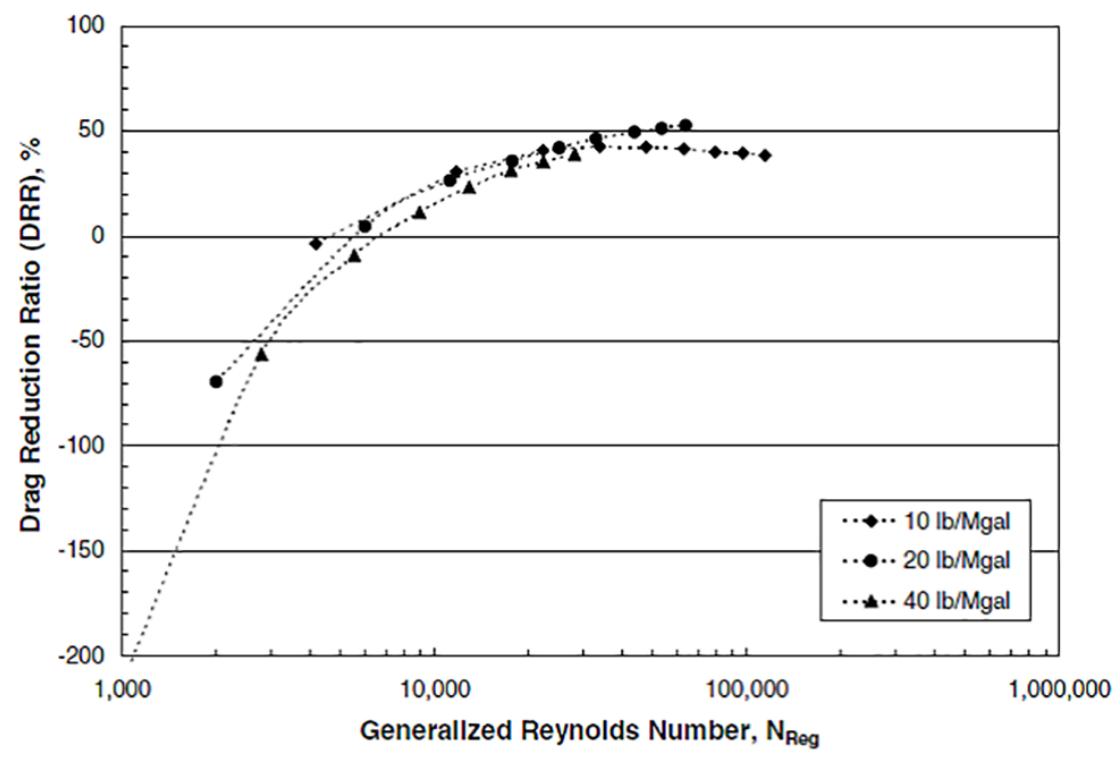

a

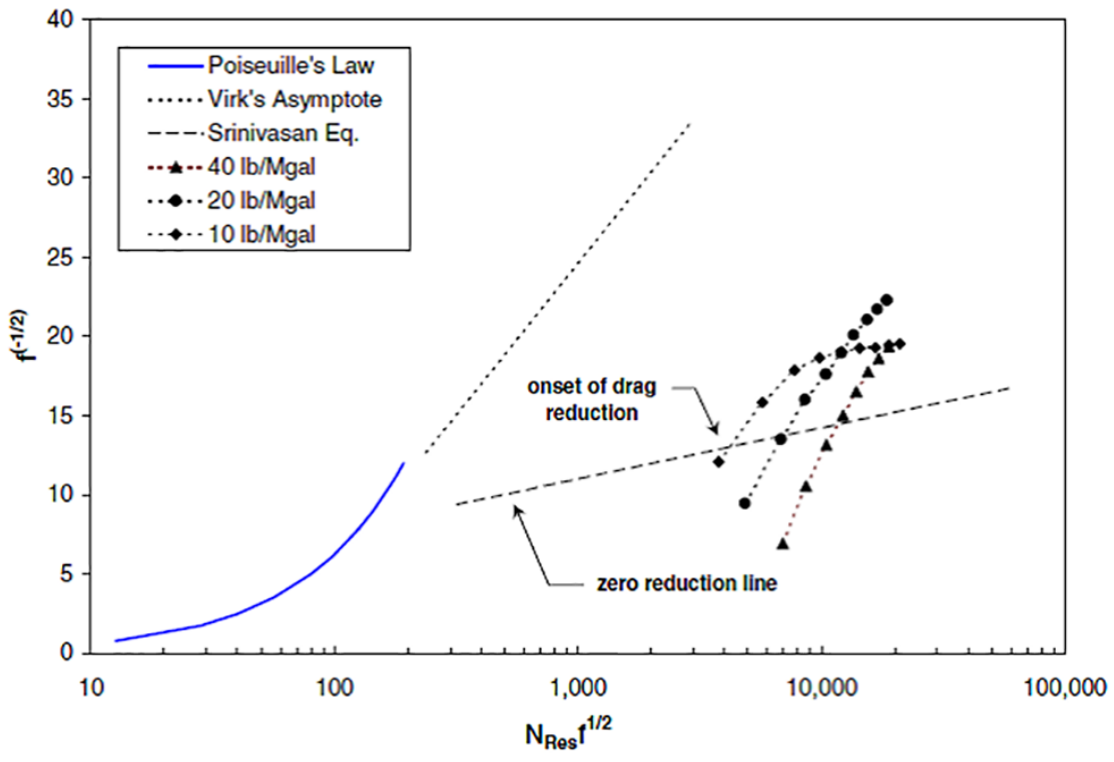

$\mathrm{b}$

Figure 6. Effect of Xanthan polymer concentration on drag reduction (2-3/8 inch. Curved pipe. (a) Drag reduction ratio versus generalised Reynolds number (b) Prandtl-Karman Coordinates. Source: [33]. 


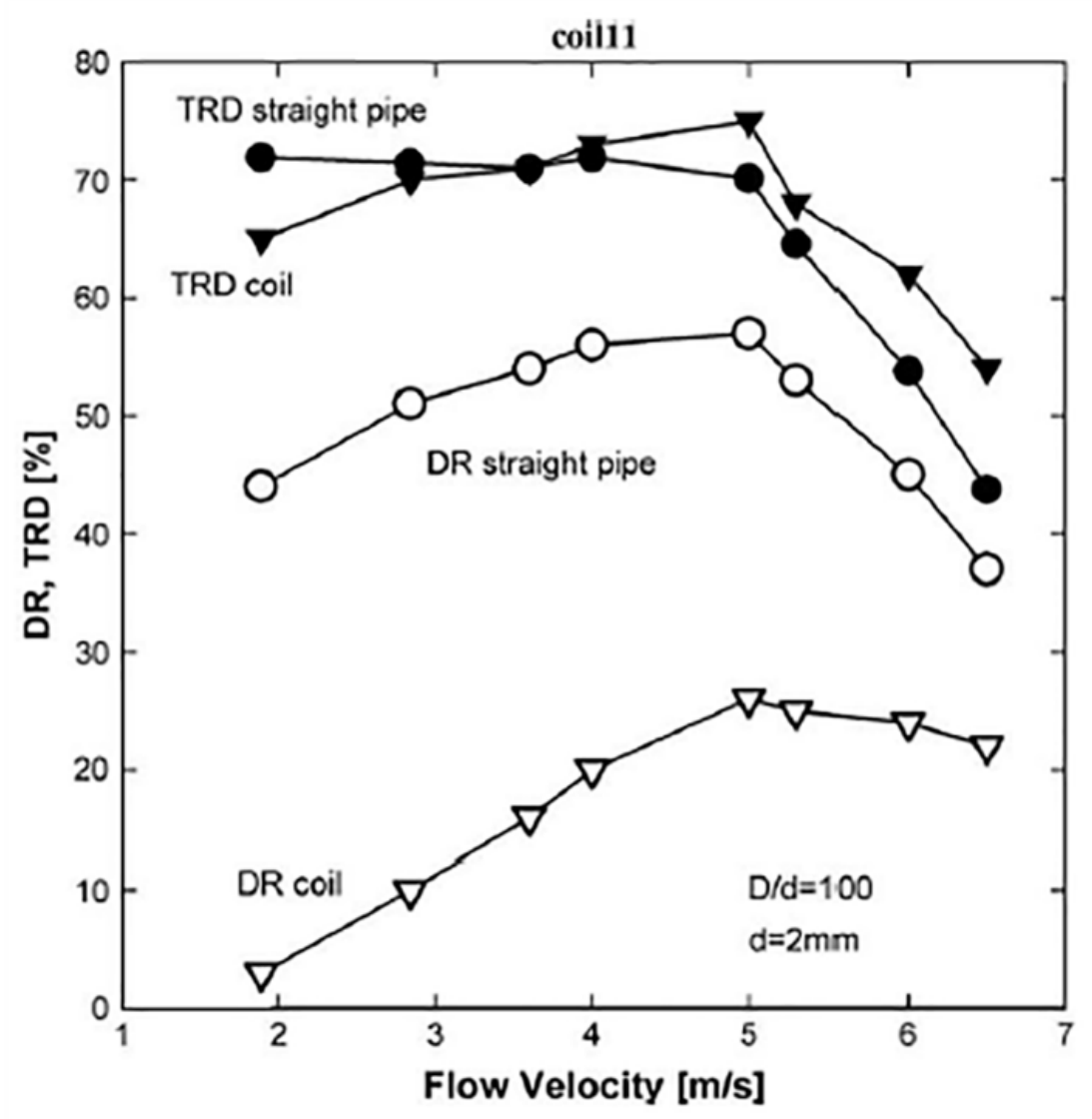

Figure 7. Drag reduction in straight and coiled pipes for flow of surfactant solution using both DR and TRD notations. Source: [10].

DR by surfactant DRAs in coiled pipes have generally been reported in the turbulent flow regime. While majority of investigations reported no DR in the laminar flow regime, a few others (e.g. Gasljevic \& Matthys 2009) reported increased drag in the laminar flow regime. The effect of surfactant DRAs on pressure drop/friction factor as reported by Aly et al. (2006) is illustrated in Fig. 5. They investigated the effect of oleyldihydroxyethylamineoxide (ODEAO) surfactant on single phase water flow in straight and coiled pipes. DR was observed in the turbulent flow regime as indicated by the reduction in friction factor with addition of the surfactant. They linked this to turbulence suppression by well-ordered network of rod-like micelles structure of the surfactant.

A few early reports on the effect of polymer DRA for flow in curves indicate a reduction in friction loss in the laminar and transition flow regime [63], [83], [84]. Their results were, however presented in terms of fluid flux (not flow resistance). They all reported increased flow rate and reduced friction loss in the laminar flow regime. It should be stated that the percentage reduction in friction loss, in the laminar flow regime, reported by some early researchers are generally very small. The reports of limited drag reduction may be explained by the interaction of drag-reducing polymer with secondary flows in the laminar flow regime in curved pipes. In more recent investigations, using advanced instrumentation to study the effects of polymer DRA on flow in curves, DR was mostly recorded in the turbulent flow regime [11], [31], [85]. 


\subsubsection{Effect of varying polymer and surfactant DRA concentration on drag reduction in coiled pipes}

The effect of concentration of drag-reducing polymer on drag reduction in hydrodynamically developed flows in coiled pipes remains unclear. Some early investigations on the effect of DRP concentration on DR (e.g. Kelkar and Mashelkar [83] - $12.5 \mathrm{~mm}$ internal diameter curved pipe/polyacrylamide polymer solution and Rao [84] - $9.35 \mathrm{~mm}$ internal diameter coil/Carbocol) reported decrease in friction factor (increase in DR) with increase in concentration. More recently, it was shown that the effect of DRP concentration on DR depends on the pipe diameter [33], [88]. For larger diameter pipes, higher concentrations resulted in lower drag reduction and even enhanced the drag at lower flow rates (Fig. 6a). In addition, higher concentration for the larger pipes delayed the onset of drag reduction. This becomes obvious when the plots are done on the Prandtl-Karman Coordinates (Fig. 6b). For smaller pipe diameter, Shah \& Zhou (2001) reported that higher concentration of polymer resulted in higher drag reduction. The effect of concentration on the onset of drag reduction for small pipes is not clear. Their plots showed no consistent patterns on the effect of DRA concentration.

Other even more recent studies did not investigate the coupled effect of concentration and pipe geometry, and the reports on the effect of DRP concentration on DR are rather inconsistent. For example Zhou et al. [31] and Shah and Zhou [33] reported higher DR when low concentration polymer was used in coiled pipes. However, reports of Shah et al. [82], Gallego and Shah [86] and Kamel [10] showed that DR in coiled pipes increased with concentration of DRA until a peak value where further increase in concentration increased drag. Shah et al. [82] used AMPS-copolymer for their study as opposed to Xanthan used by Shah \& Zhou $[30,85]$ and reported a peak concentration of $0.07 \%$ by volume polymer. This concentration was employed in subsequent works by Gallego \& Shah [86] and Kamel [10]. The optimum concentration recorded by Gallego and Shah [86] for Nalco ASP-820 and Nalco ASP-700 were $0.05 \%$ and $0.03 \%$ by volume respectively. However, the drag reduction recorded for these concentrations were very close to that of $0.07 \%$.

Reports on the effect of concentration on DR for surfactant solution flow in curves are scanty. It has been reported that below a certain surfactant concentration in the turbulent regime, no drag reduction was observed. However, beyond this concentration, the percentage drag reduction increased with increase in concentration until a value of concentration beyond which no further drag reduction was achieved (Fig. 5) [23], [73]. The reason given for this (where further increase in concentration results in no further drag reduction) is the saturation of the network structure of the rod-like micelles. Therefore, further increase in concentration was ineffective in producing additional drag reduction. Plots of Inaba et al. [70] $\left(\frac{f_{C}}{f_{\mathrm{SL}}}\right.$ versus $N_{D n^{\prime}}$ ) shows a negative drag reduction for higher surfactant concentration at low Dean number $N_{D n^{\prime}}$. However, at high Dean number it appeared that higher concentration of surfactant results in higher drag reduction. For both polymer and surfactant solution flows at fairly high Reynolds numbers, majority of reports indicate an increase in DR with increase in concentration up to an optimum concentration beyond which further increase in concentration produces no further increase in DR.

\subsubsection{Effect of fluid velocity/Reynolds number on the drag reduction in coiled pipes for polymer and surfactant DRA}

Similar to flow in straight pipes, drag reduction using polymer or surfactant DRAs in coiled pipes increases with increase in Reynolds number or flow rate (Figs. 9a and 9b) [34], [88]. In both straight and coiled pipes, the increase in drag reduction with flow rate is limited by critical shear stress above which polymer and surfactant DRAs degrade either permanently or temporarily. The difference in effectiveness (as defined by Eq. 2) of drag-reducing agent in coiled and straight pipes reduces with increase in Reynolds number (Fig. 7) [10], [34], [85]. Beyond the critical shear stress, drag reduction decreases with increase in flow rate (see Figs. 9a and 9b). Similar to observations in straight pipes, Gasljevic and Matthys [9] reported that there is no significant drag reduction in laminar flow regime in coiled pipes. 


\subsubsection{Effect of coil curvature ratio on the effectiveness of polymer and surfactant DRA}

The curvature ratio plays an important role in determining the friction losses in coils [23]. In general, when polymer DRA is used, an increase in curvature results in a delay in the onset of drag reduction (Figs. 8a and $8 \mathrm{~b}$ ). This is linked to the delay of turbulence with increase in curvature [31], [34], [85]. Shah and Zhou [33] proposed a correlation for determining the Reynolds number at the onset of drag reduction for polymer drag-reducing agents given by;

$N_{R e^{\prime}}^{*}=c_{1}-\frac{c_{2}}{\left(\frac{a}{R}\right)^{0.5}}, c_{1}=13172, c_{2}=835.33(14)$

The effectiveness of polymer drag-reducing agents generally reduces with increase in curvature (Fig. 9a and 9b) [19], [31], [85]. In the case of surfactants DRAs, there is increase in friction factor with increase in curvature ratio and this is linked to increase in the intensity of secondary flows [23], [38]. For a special case of very low Reynolds number $N_{\mathrm{Re}}<25$, Robertson and Muller [87] reported that extremely small drag reduction occurred and it increases with the curvature of the pipe. Their report requires further investigation to be validated.

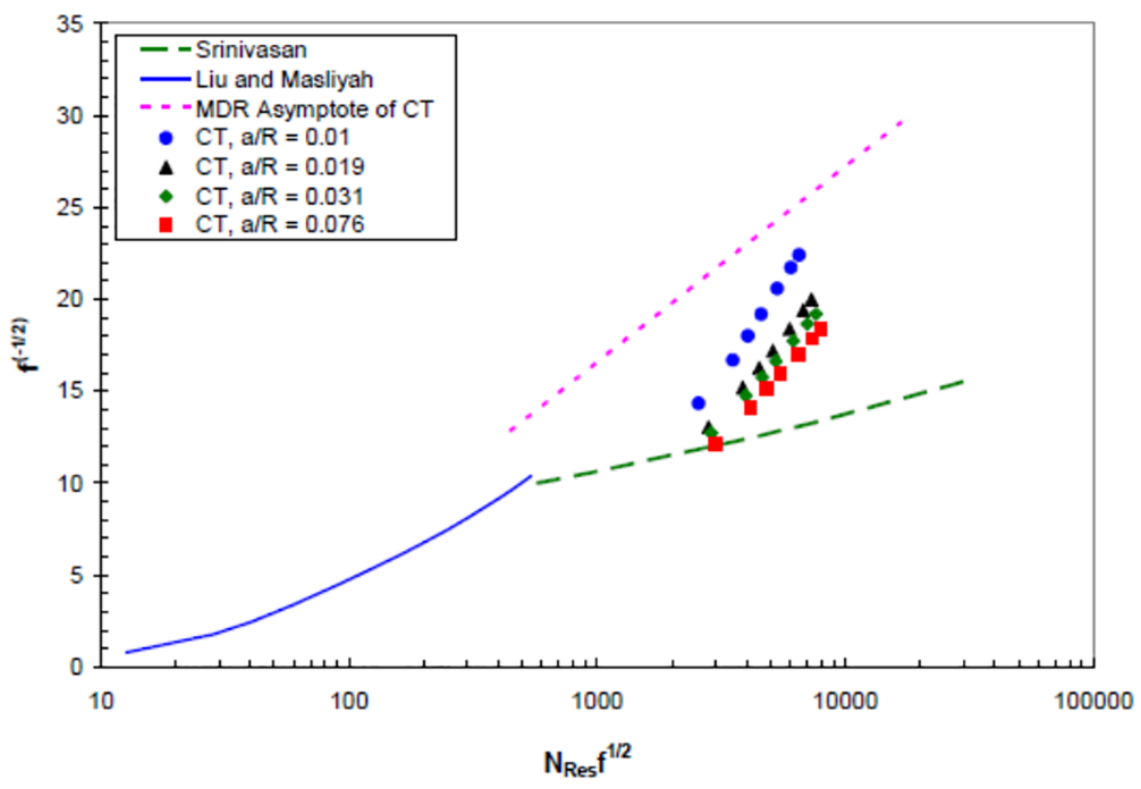




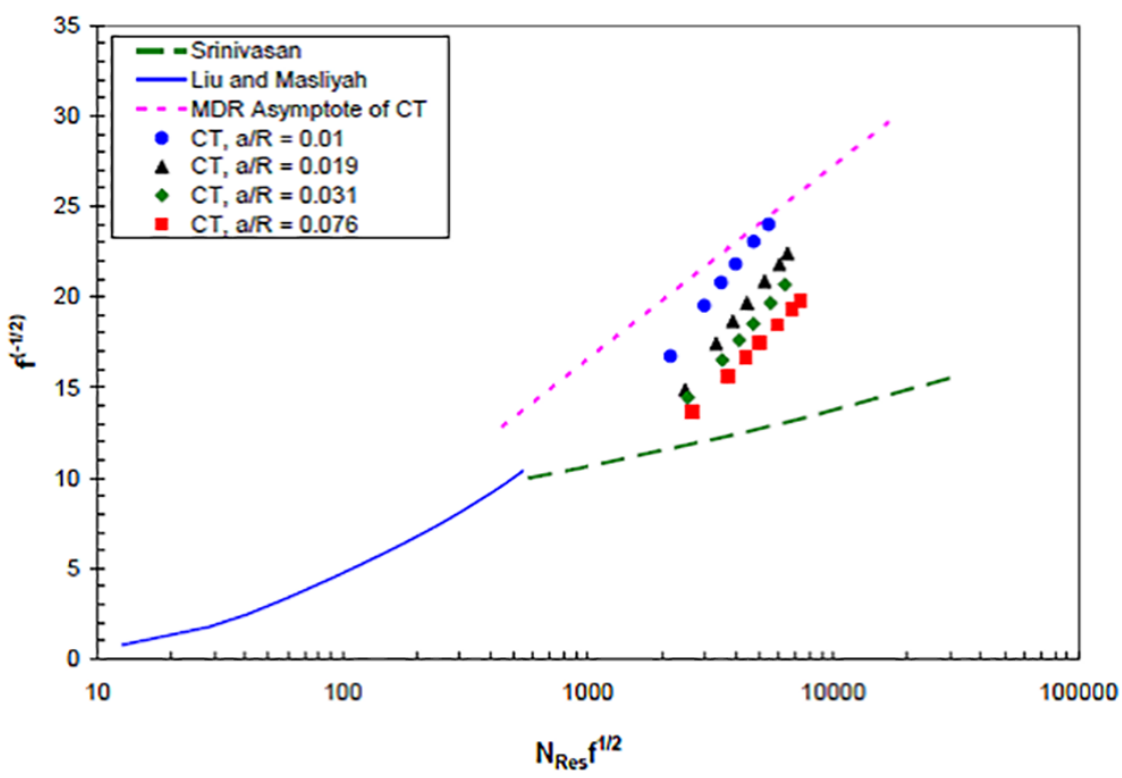

$\mathrm{b}$

Figure 8. Effect of curvature on the onset of drag reduction for (a) $10 \mathrm{lb} / \mathrm{Mgal}$ HPG in $\frac{1}{2}$-inch coiled pipe and (b) $10 \mathrm{lb} / \mathrm{Mgal}$ xanthan in $\frac{1}{2}$-in coiled pipe. Source: [19].

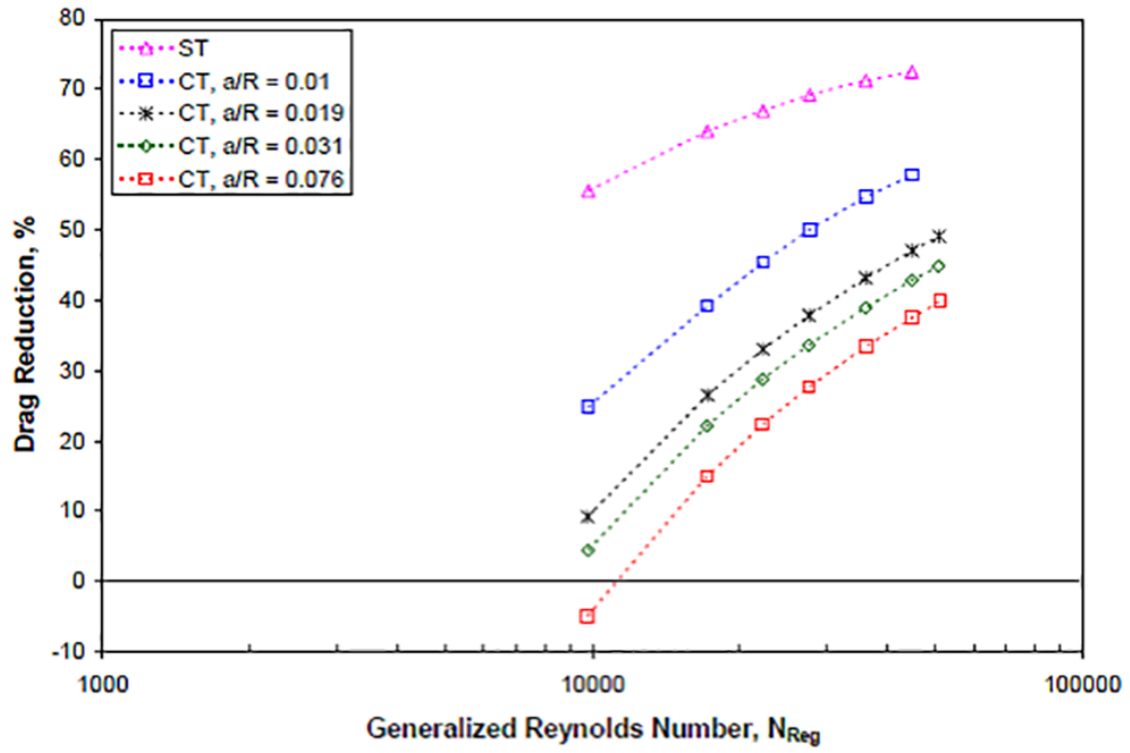

a 


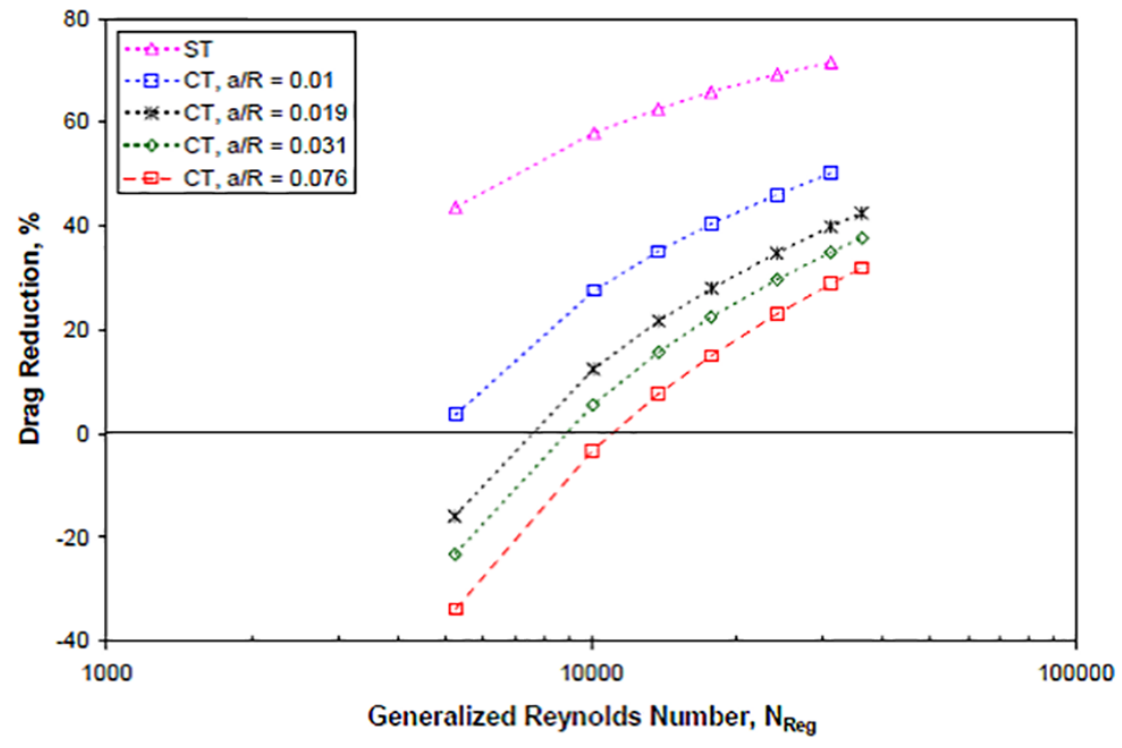

$\mathrm{b}$

Figure 9. Effect of curvature on drag reduction for (a) $10 \mathrm{lb} / \mathrm{Mgal}$ HPG in $\frac{1}{2}$-inch. coiled pipe and (b) 20 lb/Mgal HPG in $\frac{1}{2}$-inch. coiled pipe. Source: [19].

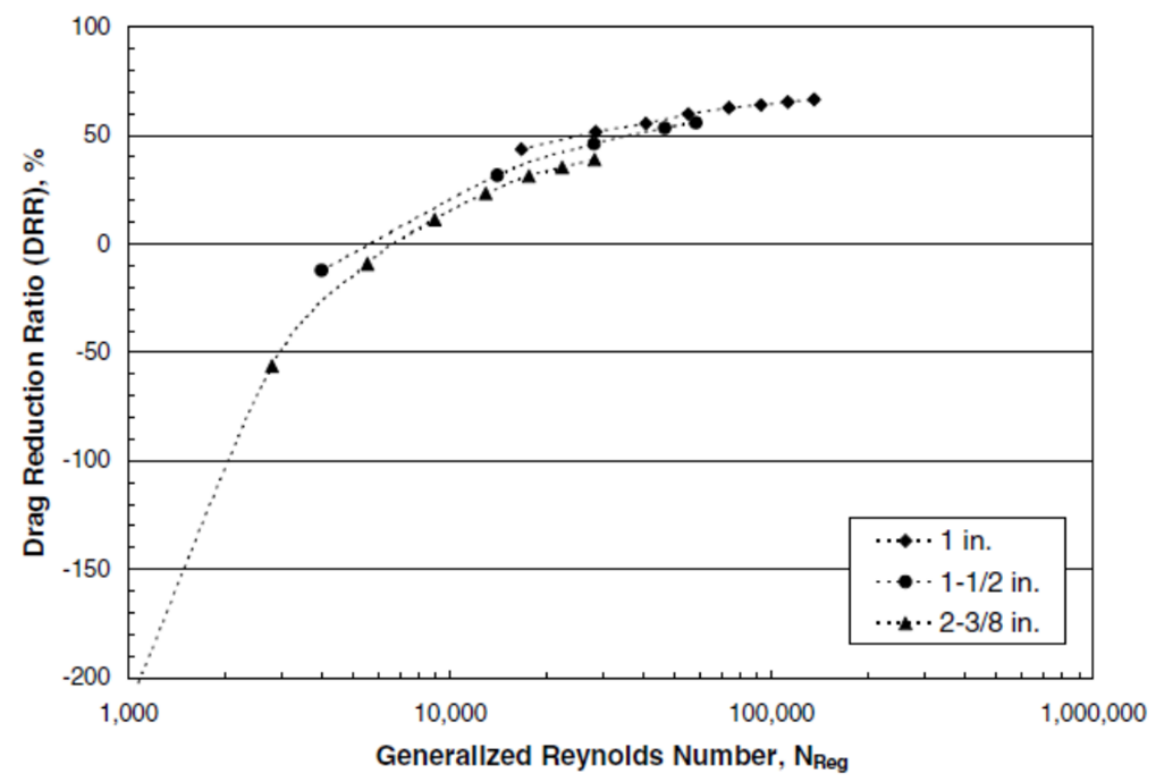

a 


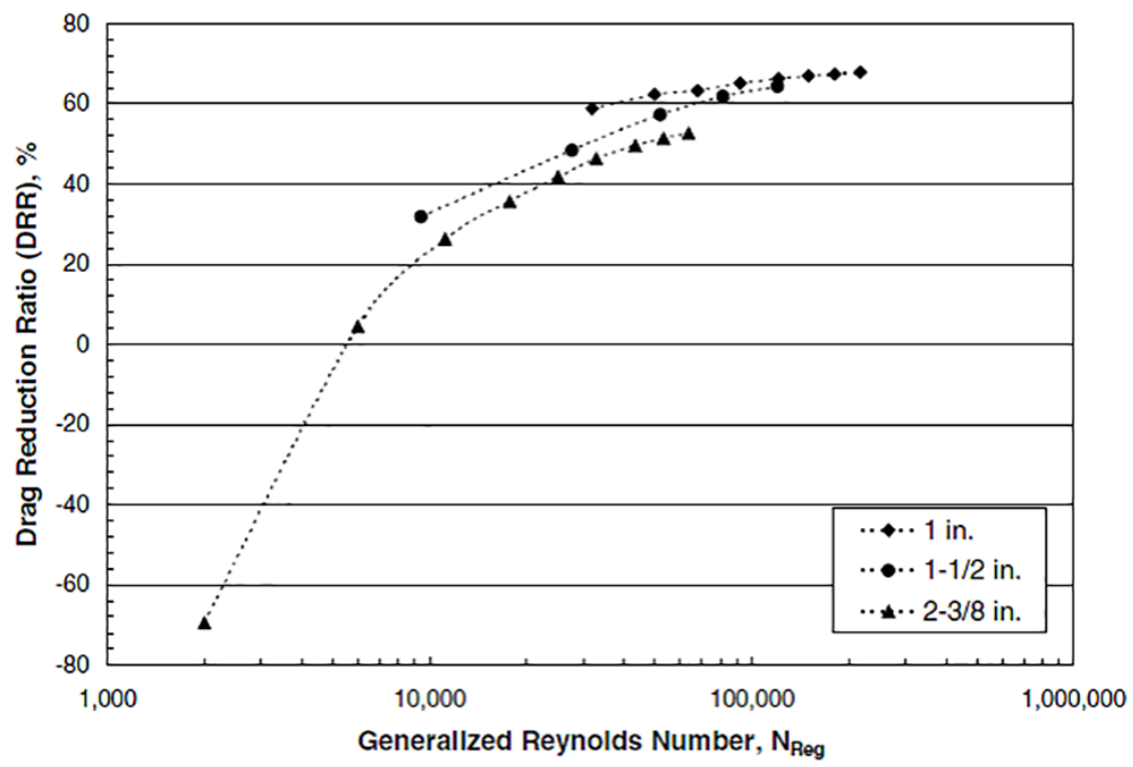

$\mathrm{b}$

Figure 10. Effect of pipe diameter on drag reduction in coiled pipe. (a) $40 \mathrm{Ib} / \mathrm{Mgal}$ xanthan fluid (b) 20 Ib/Mgal xanthan fluid. [33].

\subsubsection{Effect of pipe diameter on drag reduction in coiled pipes for flow of polymer and surfactant solutions}

The effect of diameter on the effectiveness of drag-reducing agents in curves remain unclear. For the case of polymer DRA, the effect of curvature on effectiveness of DRAs is said to be more pronounced in small diameter pipes than in larger ones [9]. Though further investigation is required, existing data show that the effectiveness of DRPs in small diameter pipes is higher than in larger ones. Coil diameter has been reported to influence the onset of drag reduction. However, for larger diameter coiled pipes the onset of drag reduction tends to a higher generalised Reynolds number [33], [88] (Fig. 10). Investigation on the effect of pipe diameter on DR by surfactant DRAs is lacking and studies in this area could throw more light on the mechanism of surfactant DR.

\subsubsection{Effect of temperature on drag reduction in coiled pipes for polymer and surfactant solutions}

Limited studies have been carried out to demonstrate the effect of temperature on the effectiveness of both polymer and surfactant DRAs. Reports show that the effect of temperature is more pronounced in straight pipes than in curves of equivalent length [11], [89]. Conflicting reports exist on the effect of temperature on effectiveness of DRPs. While Kamel [10] reported that DR was unaffected by temperature in coiled pipe flow, Gallego and Shah [86] stated that DR decreased with temperature. Data from the limited studies available for flow of surfactant solution, in both straight and coiled pipes, show that, the range and maximum values of drag reduction increased with temperature [23], [73]. This is linked to the increase in critical wall shear stress associated with increase in temperature. These tests were, however, conducted over a limited range of temperatures $\left(5-20^{\circ} \mathrm{C}\right)$ for both straight and coiled pipes. More research is required to establish the effect of a wider range of temperature for all drag reducing agents. 


\subsubsection{Effect of dissolved salts, starch or solids on drag reduction in coiled pipes for polymer and surfactant DRAs}

It has been reported that salt concentration influenced the conformation of polymer molecules [13], [76]. For the case of high salt content (typical of randomly coiled polymers chains) the DR in straight pipes occurs only after a threshold shear stress is attained. This may be associated with the uncoiling of the polymer chains of extensional flow or polymer entanglement reaching the size of eddies [91], [92]. At low concentrations in straight pipe flows, DRPs show asymptotic DR immediately after transition from laminar to turbulent flow regime [93]. It has been reported that the presence of dissolved salts in solution reduces the effectiveness of the DRA in both straight pipes and curves [9], [94]. Also adding starch to polymers is reported to have little or no effect on the frictional losses in curved pipes [9]. There is insufficient data on the effect of salts, starch and solids on drag reduction in curved pipes and so no general conclusion could be drawn.

\subsubsection{Effect of pipe roughness on drag reduction in curves for polymer and surfactant solutions}

Pipe roughness is expected to have appreciate effect on DR since it is likely to affect both velocity fluctuations and degradation of polymer and surfactant DRAs. It has been reported that the mechanism that sustains turbulence in smooth and rough pipes are quite different [95]. Due to the limited studies in this area, the effect of pipe roughness on DR it remains unclear. In the case of straight pipe flow of DRPs, Karami and Mowla [93] reported increased DR with increase in pipe roughness, while in a rectangular open channel Petrie et al. [94] observed a decrease in DR with pipe roughness. This discrepancy is not entirely surprising. The increase in percentage DR with pipe roughness reported by Karami and Mowla [93] could be explained by increased velocity fluctuation and turbulent intensities with increase in pipe roughness. In smooth pipe flows increase Reynolds number is often associated with increase in velocity fluctuation and turbulent intensities. It has been reported that, below critical wall shear, percentage DR increase with Reynolds number [34], [88]. Therefore, one would expect an increase in percentage DR with increase in velocity fluctuation and turbulent intensities associated with increased pipe roughness. On the other hand, increased pipe roughness could also increase the rate of polymer degradation within the channel. The implication of this would be reduced percentage DR with increase in pipe roughness, especially for high Reynolds number flows. Since increased turbulent intensities/velocity fluctuation and increased DRA degradation could result from flow over rough surfaces, the dominant of the two would most likely determine whether there is increased or reduced DR with roughness. Gallego and Shah [86] reported that, for the flow of DRPs, the effect of pipe roughness was more pronounced in curved pipes than in straight pipes. Their plots showed that pipe roughness results in decrease in DR in coiled pipes. This might be associated with increased shear in curved pipe flow when compared to straight pipe flow which in turn results in degradation of polymer chains.

\subsection{Effect of polymer and surfactant DRAs on pressure drop for single phase liquid flows in bends}

Flow through bends exhibits a more complex geometry than flow in coiled pipes. This is because of entry and separation effects coupled with the idealised flow similar to that in coiled pipes. The disturbance generated at the bends, increases the downstream flow redevelopment length. The redevelopment for surfactant solutions, for example, has been reported to be slower than that of water [10]. The redevelopment is even slower at higher velocities (Fig. 11). This could be the result of higher drag and heat transfer reductions associated with higher velocities. The slower redevelopment for flow of surfactants compared to water introduces added drag which counters its drag-reducing effects on the flow [10]. This additional drag effect is linked to flow separation. It has been reported that, for flow of surfactant flow through threaded elbows there is significant drag reduction upstream and downstream (after full development) of the elbow but not in the elbow region of undeveloped flow itself [10]. The report indicates that, although there is drag reduction in the bend, the overall effect of flow redevelopment is overbearing on the drag reduction. One may deduce from the work of Gasljevic and Matthys [9] that, the difference in drag for surfactants in the elbow is linked to the effect of the surfactant on flow separation and reattachment. Thus, if the surfactant reduces turbulence, it may hinder reattachment and energy transfer to the wake. There is however insufficient data to fully explain the effect 
of surfactant DRAs on pressure drop in bends. At the time of this report, studies of polymer DRAs in bends could not be found in open literature. However, since only small concentrations (compared to surfactants) of DRPs are required for DR, it should be expected that flow redevelopment for DRPs solution in bends would approach that of the solvent. If this is the case, below the critical wall shear, addition of DRP is likely to yield significant DR for single phase liquid flows in bends. In addition, since surfactants undergo temporary degradation at high shear, flow rate control can be used as a means of optimising drag-reducing effects of surfactants in and around bends. Experimental investigation is currently under way by the authors to gain more insight into the effect of DRPs on flows in bends.

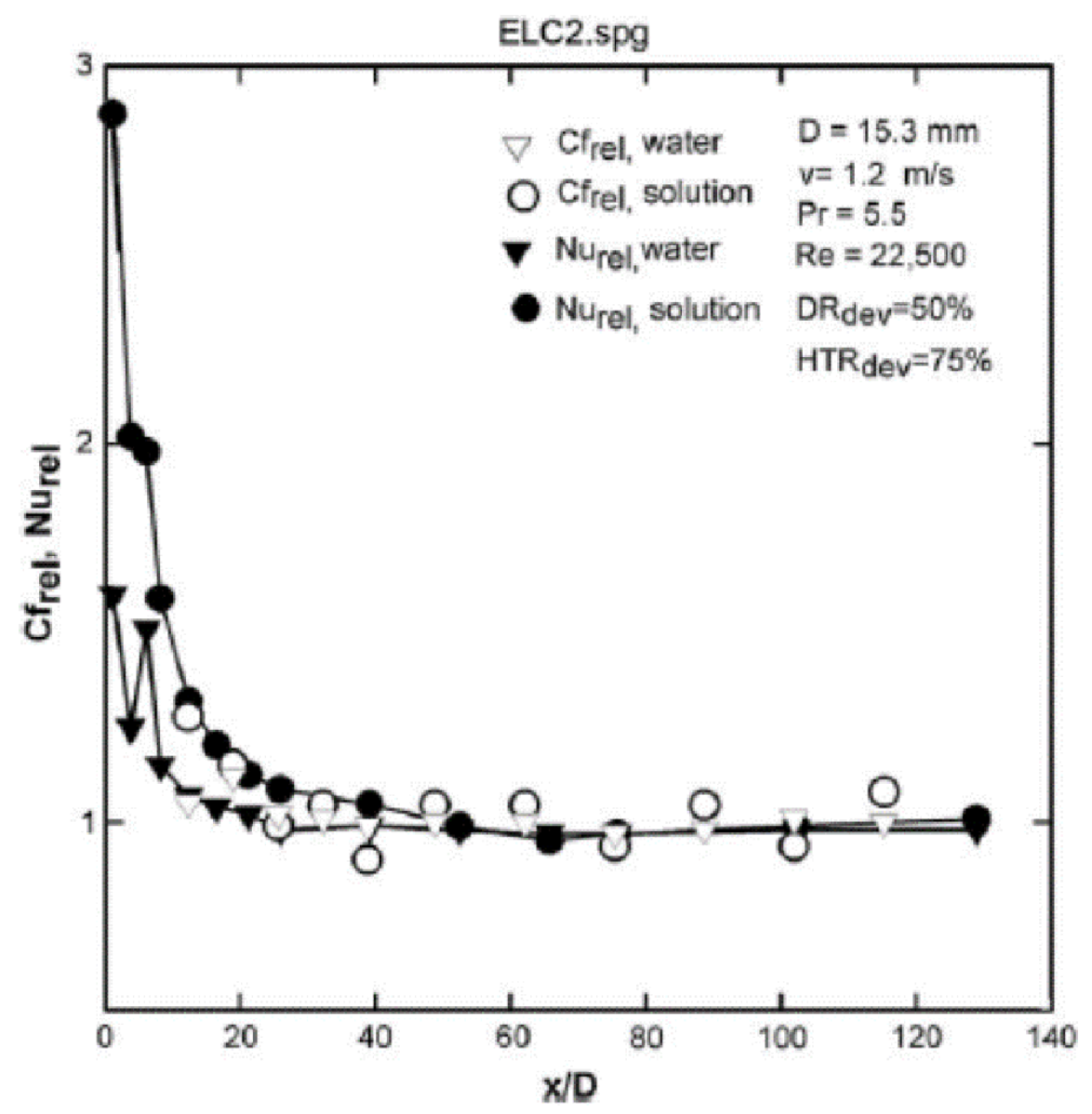

a 


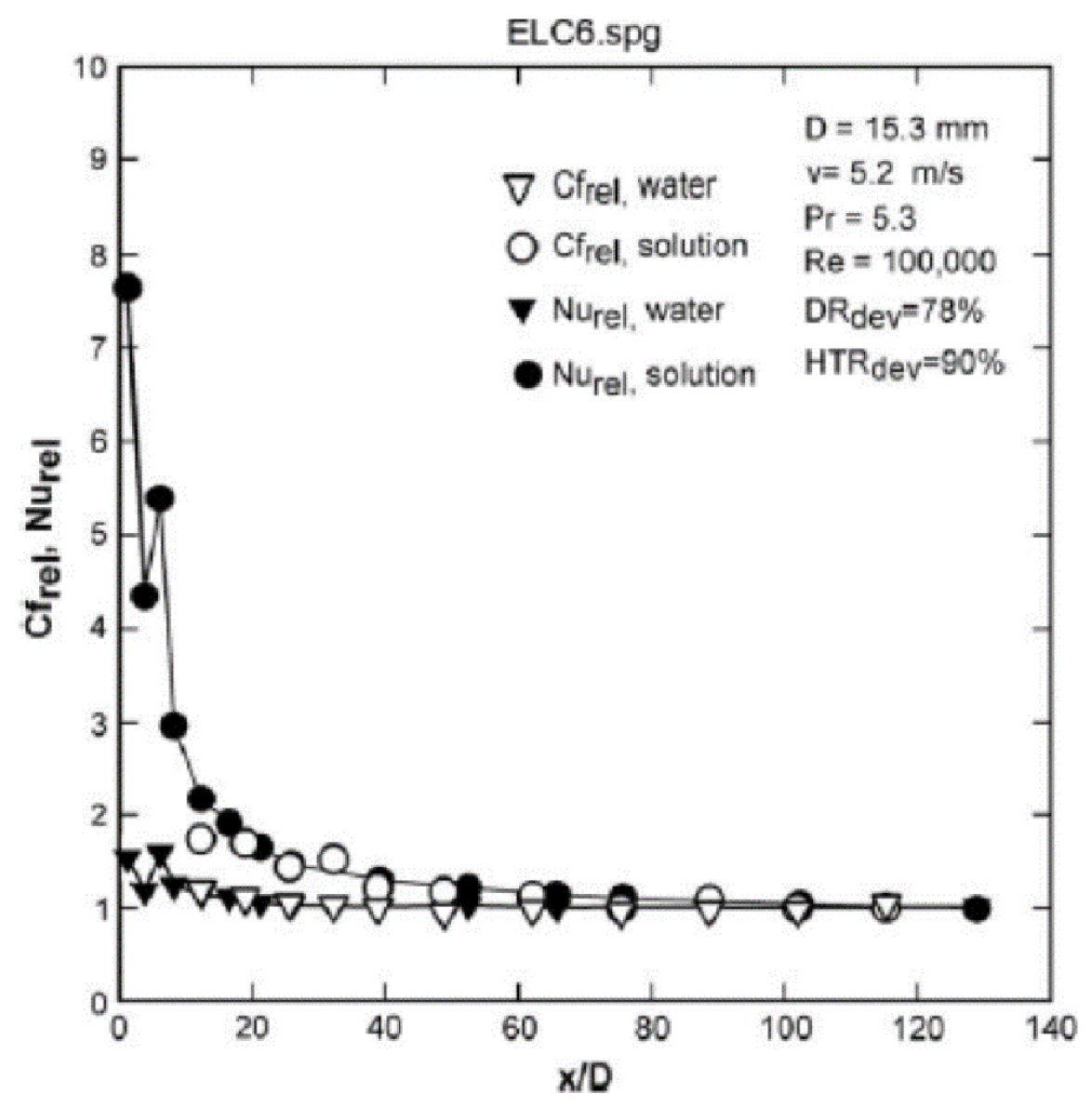

$\mathrm{b}$

Figure 11. Nusselt number and friction coefficient at the downstream section of 1.2" elbow relative to their values at $x / D$ (fully redeveloped flow). (a) velocity $=1.2 \mathrm{~m} \mathrm{~s}^{-1}$ (b) velocity $=5.2 \mathrm{~m} \mathrm{~s}^{-1}$. Solution: $2300 \mathrm{ppm}$ ETHOQUAD T13 + 2000 ppm NaSal in a molar ratio to surfactant of 2.5:1. Source: [10]. 


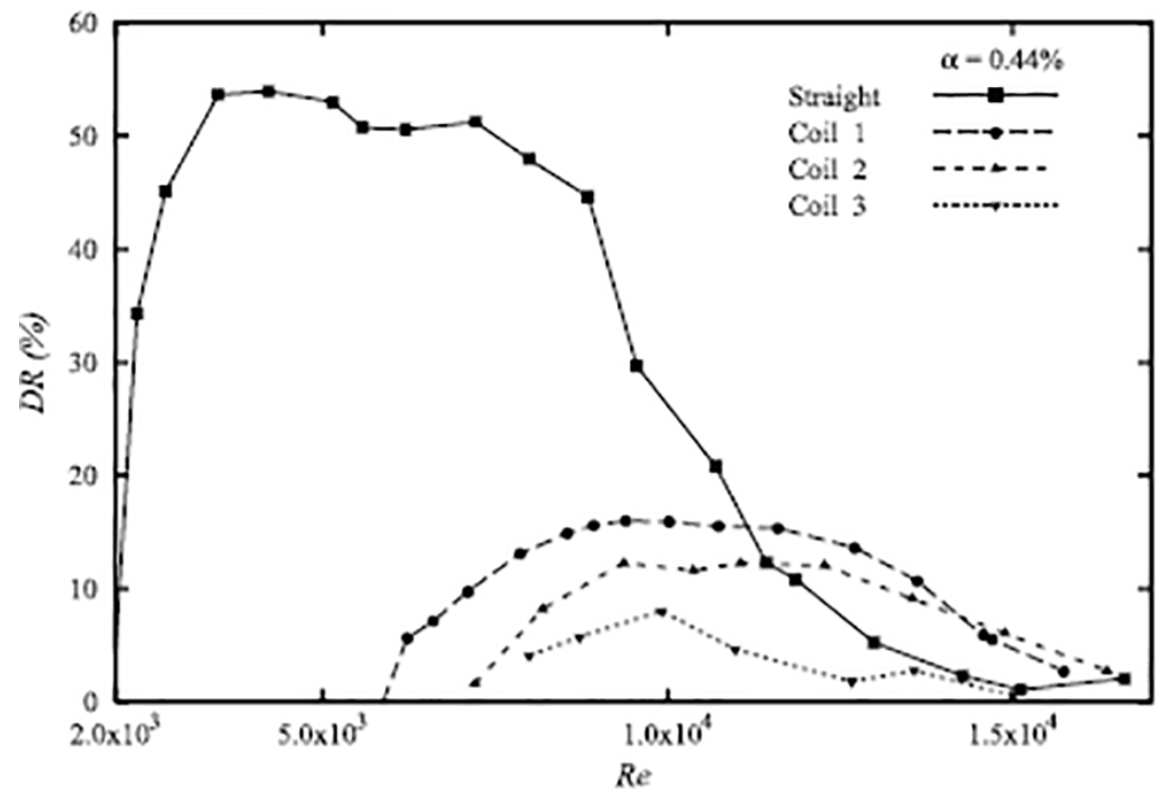

a

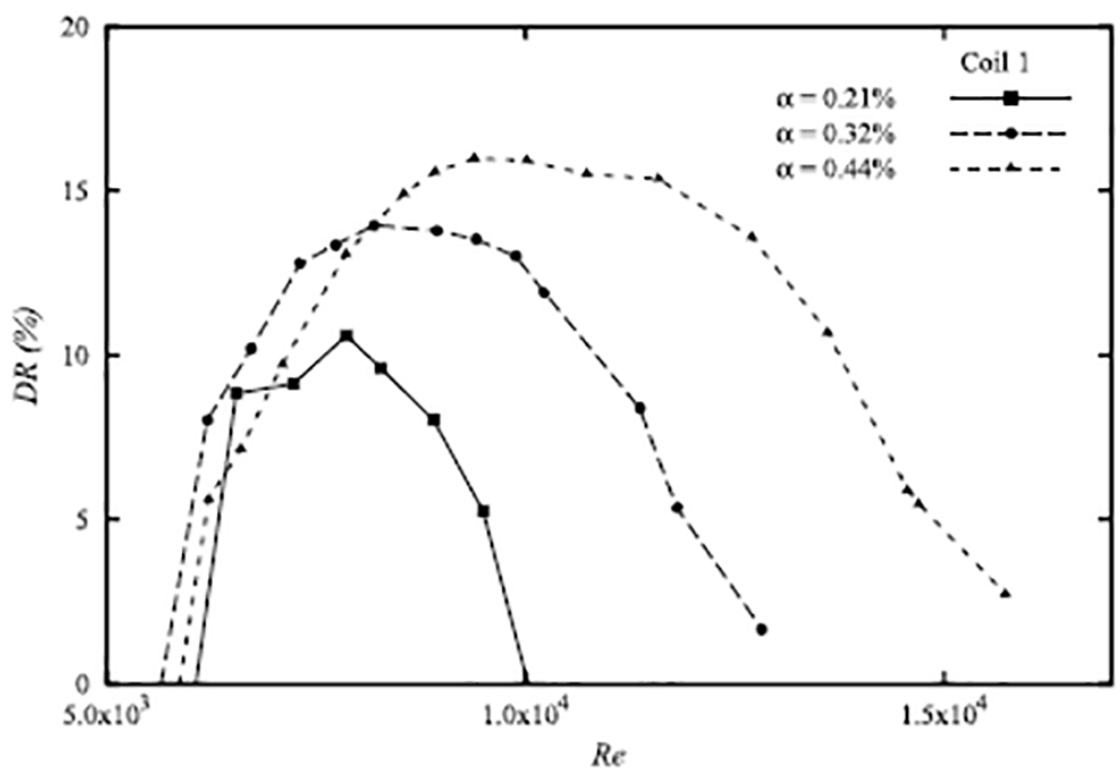

$\mathrm{b}$

Figure 12. Drag reduction versus Reynolds for flow of water in helical coils with the injection of microbubbles. $\alpha$ is the fraction of air in the coil. (a) effect of curvature ratio (b) effect of air fraction. Source: $[78]$. 


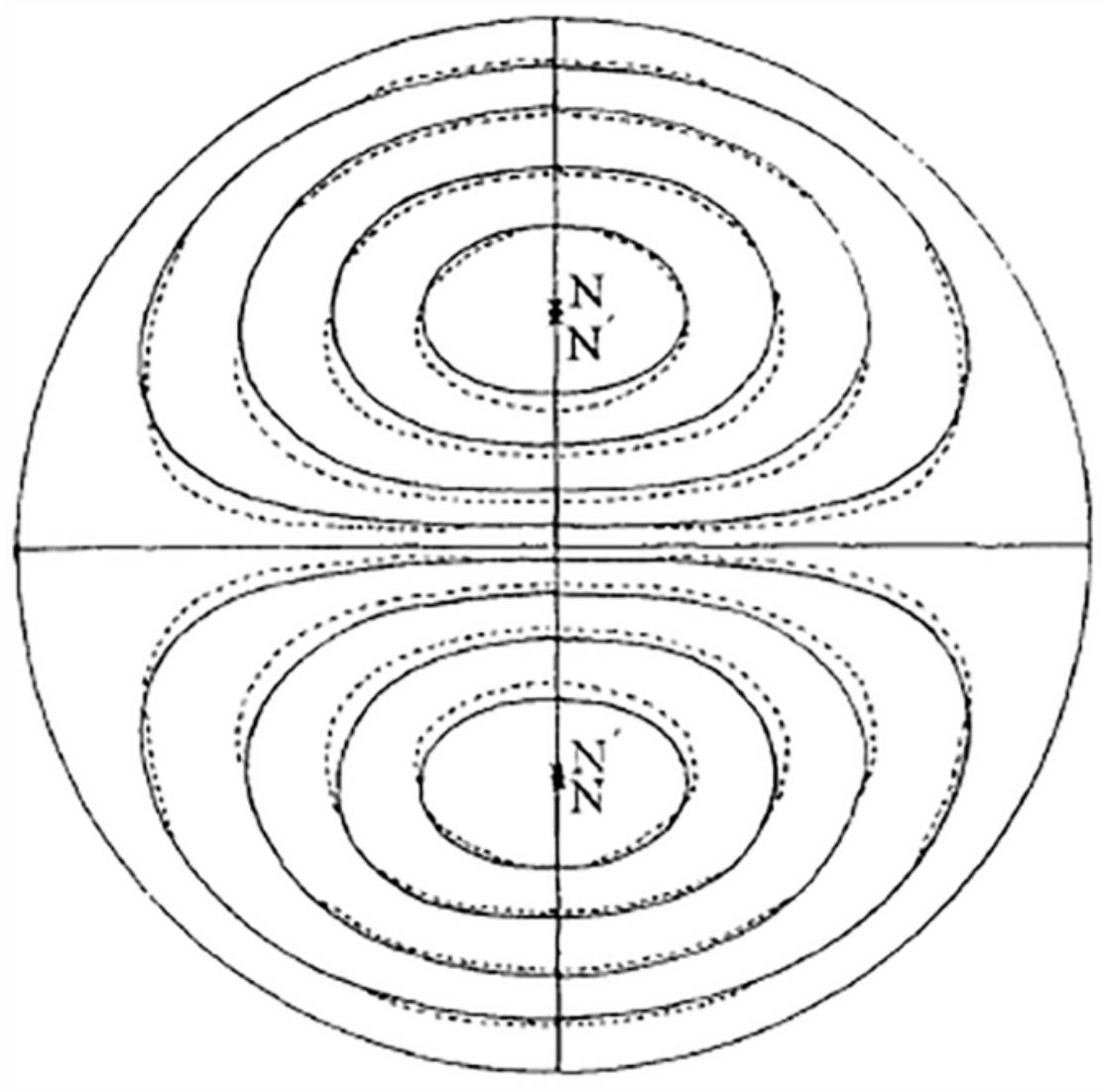




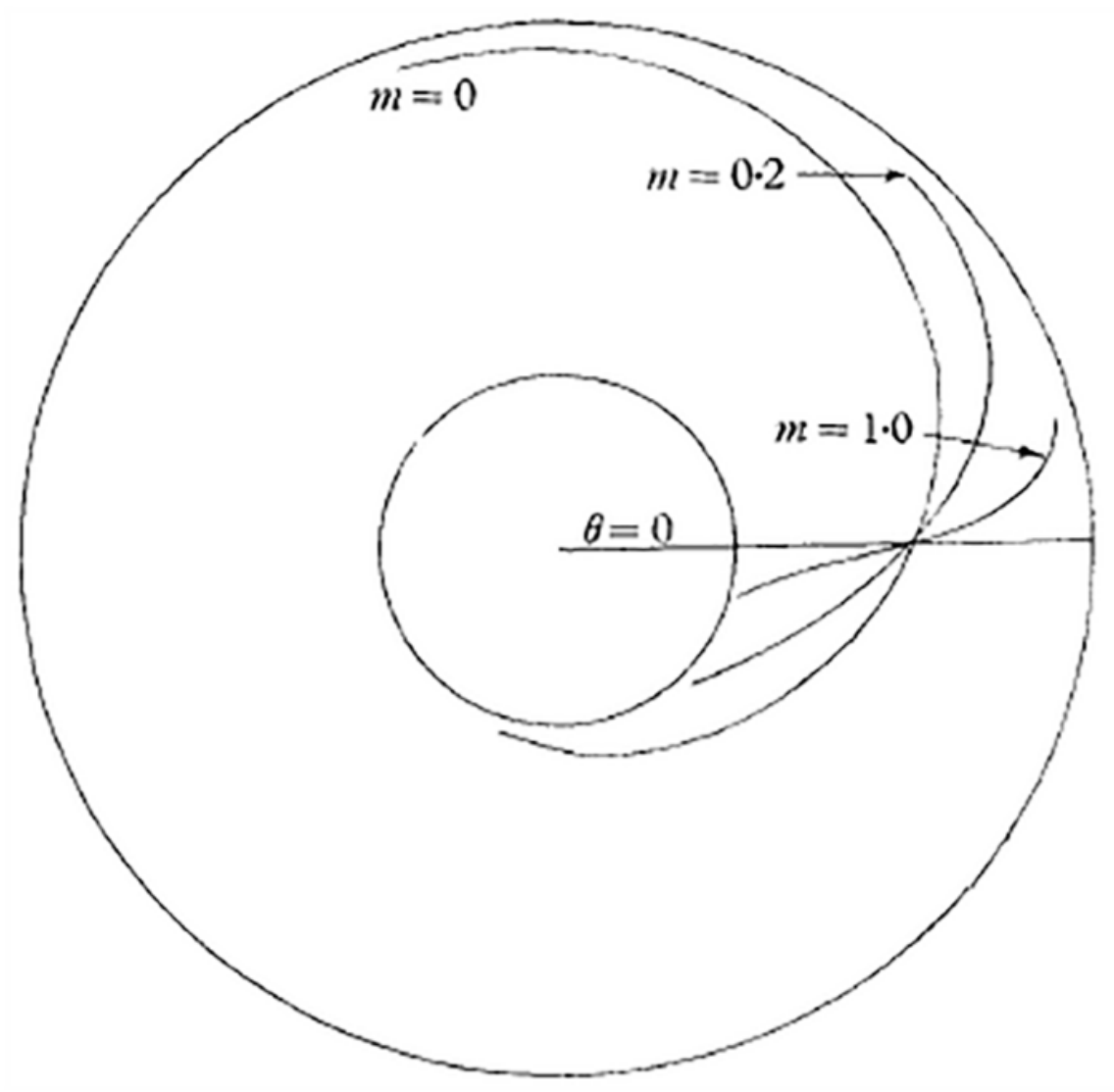

$\mathrm{a} b$

Figure 13. a. Particle paths projected on the cross-section of flow for $m=1$ (full line) and $m=0$ (dash line). $N$ and $N^{\prime}$ represent neutral points for $m=1$ and $m=0$ respectively. Source:[98], $b$. Paths of particles in the central plane for varying $m$. Source: [98].

\subsubsection{Effect of DRAs concentration on pressure drop in bends for polymer and surfactant solutions}

No comprehensive work exists in open literature that investigates the effect of polymer and surfactant concentration on drag reduction in bends. Munekata et al. [95] investigated the flow of surfactant solution $(\mathrm{CTAB})$ in $90^{\circ}$ square-cross-section bend where reduced drag reduction as well as delayed onset of drag reduction with increase in concentration was reported. Only two concentrations were tested and so a reasonable conclusion cannot be drawn. Their plots showed reduced critical Reynolds number (or flow velocity) with decrease in concentration. The effect of concentration of drag-reducing agents for flow in bends such as $45^{\circ}$ and $180^{\circ}$ remain unclear. Notwithstanding the limited literature in this area, DRA concentration is expected to impact DR efficiency since concentration can influence flow redevelopment and the overall ability of the DRA to supress turbulence [10].

\subsubsection{Effect of curvature on effectiveness of DRAs in bends for flow of polymer and surfactant solutions}

Bend angles and curvatures is known to influence centrifugal forces in the bends and consequently affects fluid redistribution in and around the bend. While in coils, increased curvature delays the unset of turbulence and supresses turbulence, in the case of bends, increased curvature may result in increased flow fluctuations, even 
under laminar flow conditions. There are contracting reports from the limited studies that have been carried out to investigate the effect of curvature ratio hence, its effect remains unclear. For low Reynolds number flows, Jones and Davies [96] reported that the effect of curvature on drag reduction is negligible. Nonetheless, the observed drag is higher than that which occurred in straight pipes. Yokoyama and Tomita [97] studied the flow of polyethylene-oxide in a $360^{\circ}$ bend of varying curvature ratios. They recorded a decrease in drag reduction with increase in curvature ratio. The drag reduction recorded was predominant at high Reynolds numbers. Only three curvatures were tested and so the effect of curvatures on the effectiveness of dragreducing agents in $360^{\circ}$ bends was inconclusive. In the knowledge of the Authors, the effect of curvature ratio for flow in $45^{\circ}, 90^{\circ}$ and $180^{\circ}$ bends had not been reported in open literature. Another area of interest is in determining the effect of pipe diameter on drag reduction in elbows. This is because; the effect of flow separation is expected to reduce with increase in pipe diameter.

\subsection{Effect of micro-bubble injection on pressure drop for single phase liquid flows in coiled pipes}

Application of micro-bubbles for DR in curved pipe flows have received little scholarly attention and the effect of micro-bubbles on pressure losses as well as the mechanism of micro-bubble DR remain unclear. It was highlighted earlier that, the action of micro-bubbles on turbulent flows is similar, in a number of ways, to that of polymers and surfactant DRAs. To this end, it is expected that micro-bubbles will result in significant DR in curved pipe flows. The application of micro-bubbles for drag reduction in helical coils was first carried out by Shatat et al. [75] and Shatat et al. [98] using hydrocyclone effect to generate micro-bubbles. Their investigation involved three helical coils of curvature ratios $0.025,0.05$ and 0.1 . They reported that, though there was significant drag reduction in helical coils by injection of micro-bubbles, this drag reduction is less than that in straight pipes under similar conditions of flow. The reduced drag reduction in helical coils is linked to centrifugal forces (resulting in suppressed turbulence) associated with the flow. Though the theories for micro-bubble drag reduction in helical coils are in agreement with existing micro-bubble DR theories for other geometries, further research is needed to establish these theories. The effect of various parameters such as pipe geometry, micro-bubble fraction, flow rate and micro-bubble size remains unclear due to the limited research in this area. Based on the limited data available, only a brief outline of the effect of these parameters is presented in this review.

\subsubsection{Effect of curvature ratio on micro-bubble drag reduction in coiled pipes}

Fig. 12a gives an illustration of the effect of curvature on effectiveness of micro-bubble drag reduction in helical coils. It can be observed that increase in curvature resulted in decrease in drag reduction as well as a shift of both the onset of drag reduction and maximum drag reduction to higher values of Reynolds number. The figure also shows higher drag reduction in straight pipes compared to helical coils. Though there is limited data on the effect of curvature, two important hydrodynamics properties may play important roles: first, unlike flows of polymer and surfactant solutions in curved pipes, gravity/centrifugal forces may result in significant phase separation (micro-bubble and liquid phases) for the case of micro-bubble DR. If this occurs, the concentration distribution of micro-bubbles (particularly in the buffer region where it is most effective) becomes inhomogeneous and this is likely to reduce DR efficiency; second, the curvature effects in coils is expected to suppress turbulence and thus it should be expected that the percentage DR is affected by the degree of curvature. Further study is, however, required to fully investigate the effect of curvature ratio on drag reduction in curves.

\subsubsection{Effect of air micro-bubble fraction on drag reduction in curved pipes}

The effect of micro-bubble fraction is illustrated in Fig. 12b. It can be seen that the effect of air fraction on the onset of drag reduction is insignificant. However, the air fraction has a profound effect on the percentage drag reduction and the range of Reynolds numbers over which drag reduction occurs. In general, the 
percentage drag reduction increased with increase in air fraction. Again, additional data is needed in order to understand the effect of micro-bubble fraction on drag reduction since very scanty reports are available.

\subsubsection{Effect of flow rate on micro-bubble drag reduction in curved pipes}

Similar to flow of polymer and surfactant solutions in curved pipes, where DR is reported predominantly in the turbulent flow regime, the limited reports on the application of micro-bubbles in curved pipe DR also report it in the turbulent flow regime. Since the degree of turbulence increases with increase in flow rate, it is expected that flow rate will affect the efficiency of micro-bubble DR. It can be seen from Figs. 12a and $12 \mathrm{~b}$ that drag reduction occurs above a critical Reynolds number and increases with Reynolds number until a maximum drag reduction is achieved. Further increase in Reynolds number decreases the drag reduction. At very high Reynolds numbers, there is increased centrifugal forces [103] resulting in lower shear stress near the inner wall and higher shear stress in the region close to the outer wall. The implication of this is the uneven distribution of air bubbles and thus reduced drag reduction.

\subsubsection{Effect of micro-bubble size on drag reduction in curves and bends}

In the knowledge of the Authors, no published research is available that investigates the effect of microbubble size on DR in curved pipes. There is therefore need for more research to enhance understanding of any possible effect of micro-bubble size on DR [28]. In the application of micro-bubbles as drag-reducing agents for straight channel flow, conflicting reports exist on its effect on DR. It suffices to say, however, that bubble behaviour is size dependent, thus DR is expected to be influenced by micro-bubble size. In general, small sized bubbles will be better retained in the liquid under the action of centrifugal forces. Hence, it would be expected that the smaller the size of the bubbles the more effective it'll be as a DRA.

\subsection{Effect of polymer and surfactant DRAs on fluid flux in curved pipes}

A number of early researchers chose to present their results in terms of flow rates rather than drag. The limited studies in this area have focussed on the application of polymer DRAs in curved pipe flows. There appears to be an agreement among the limited reports that addition of DRPs results in increased flow rate particularly at low and moderate Dean numbers [63], [100].

Barnes and Walters [60] reported that, for fully developed turbulent flows in curved pipes, there is decrease in flow rate after adding polymer. It was suggested that the suppression of turbulence may have an adverse effect on the flow rate at high Reynolds numbers. Given that a number of recent studies have reported DR in the turbulent flow regime, it is possible that the polymer used in that study has degraded at the turbulent flow conditions studied. They also reported an increase in flow rate with increase in polymer concentration and a negligible influence of pipe curvature on the effectiveness of the DRPs in the laminar and transition flow regimes. Though further research is required to understand the effect of fluid characteristics and pipe geometry on the flow rate of DRAs, the limited research available suggest that flow rates would increase in the region of DR.

\subsection{Secondary flow in bends and curves}

The secondary flow observed for the flow of Newtonian fluids in bends and curves results from centrifugal forces associated with such flow. The secondary flow of spiral form superimposes on the axial primary flow and there is also reduction in flow rate as a result of higher dissipation resulting from secondary flow compared to primary flow. The maximum axial velocity in curves and bends is shifted to the outer side of the curve. As Dean number increases the secondary flow become more confined to a thin area near the pipe wall [19]. At higher Reynolds number additional pairs of vortices appear and multiple solutions exist [104]. 


\subsubsection{Effect of DRAs on secondary flow for single phase flows in curves}

It has been suggested that drag-reducing agents would have an effect on secondary flows [10]. At high flow rates, the secondary flow field can be categorised into two regions. These are the shear free mid-region and the offside boundary layer region [105]. The non-Newtonian characteristic of fluid changes the thickness of the shedding layer. For pseudo-plastic fluids the shedding layer becomes thicker, whereas for dilatant fluid flow it is thinner than that of Newtonian fluids. This thickening or thinning effect may, to a small or large extent, alter the secondary flow. Fig. 13a shows the paths of fluid particles projected on the cross section of the pipe. The extremes of $m=1$ and $m=0$ represents viscoelastic and Newtonian viscous liquids respectively. It is seen from the figure that, the effect of elasticity (measured roughly by $m$ ) on the projected streamlines is small. However, the neutral point for the viscoelastic liquid is slightly nearer to the outer edge of the pipe compared to that for the Newtonian liquid.

The elasticity of the liquid has a profound effect on the pitch of the spirals in which the liquid particles move along the central plane (Fig. 13b). Fig. 13b shows that a decrease in $m$ leads to a major increase in the curvature of the streamlines in the central plane. The main effect of elasticity on the flow of viscoelastic liquids through a curved pipe is to decrease the curvature of the streamlines in the central plane and to increase the fluid flux through the pipe [63], [98].

For a third-order fluid (see Coleman and Noll [103]), Jones [80] presented correlation (Eq. 15) for the streamline function, which describes the secondary flow in the cross-section of curved pipes.

$\Psi=\frac{2 L \alpha_{1}}{\rho \alpha}\left[\left(\frac{1}{144}+\frac{\alpha_{3}^{\prime}}{48}\right) r_{1}-\left(\frac{1}{64}+\frac{\alpha_{3}^{\prime}}{24}\right) r_{1}^{3}+\left(\frac{1}{96}+\frac{\alpha_{3}^{\prime}}{48}\right) r_{1}^{5}-\frac{r_{1}^{7}}{576}\right] \cos \cos \alpha(15)$

Eq. 15 indicates that, for third-order fluids, the non-Newtonian effect on secondary flow streamline could be associated mainly to the elastic behaviour $\left(\alpha_{3}\right)$ of the fluid.

It has also been suggested that an analogy existed between the counter-rotating secondary flow vortex superimposed on the primary flow in curved pipes and the vortex pair at the near wall region of turbulent shear flow in straight pipes [65]. Since drag reduction is a phenomenon of the near wall region where the flow is primarily a shear flow, it is suggested that any mechanism that results in this phenomenon would also affect the secondary flow in curved pipes, at least in the laminar flow regime. It should be stated here that this assumption relies on the notion that secondary flow, like turbulent flow, is dissipative. Though a few other studies [87], [107], [108] made brief mention of the effects of DRAs on secondary flows, there is insufficient data from which concrete conclusions can be drawn.

\subsubsection{Effect of DRAs on secondary flow in bends}

There are very few studies on the effect of DRAs on secondary flows in bends and though the limited reports agree that such effects exist, there is no clarity on whether DRAs suppresses or enhances secondary flows. In the study carried out by Jones and Davies [96] using very dilute polyacrylamide and Kezan solution, the onset of non-Newtonian effects was around Dean number of 300. This is the region where secondary flow with a Newtonian fluid is sufficiently strong enough to cause appreciable deviation from Poiseuille flow . Munekata et al. [95] in their study of viscoelastic fluid flow in square-section elbow bends suggested that centrifugal effects are suppressed by viscoelastic effect of the fluid flow. They reported that secondary flow for Newtonian fluids increases gradually downstream while for viscoelastic fluids it decreases slightly resulting in DR. Their result was not corroborated by any other research findings, and further study is therefore required.

\subsection{Flow transition and critical Reynolds number in bends and curves of cir- cular cross-section}

Studies show that, flow transition in curved pipes occurs at much higher Reynolds number than in straight pipes. There is also delayed onset of turbulence with increase in curvature. Taylor [106] in one of the 
early researches in this area showed that streamline motion persisted to Reynolds number of about 6000 in curved pipe of $\frac{\mathrm{a}}{R}=\frac{1}{18}$. The mechanism by which turbulence is produced in curved flow varies with the location in the curves [110]. Turbulence near the inner wall results from gradual superposition of higher order frequencies on the fundamental frequency. On the other hand, turbulence, near the outer wall, results from high frequency bursts near the outer wall. The sinusoidal oscillations near the inner wall always precedes the turbulent bursts [19]. The transition region for flow of Newtonian fluids in straight pipes is associated with violent flashes which is not the case in curved pipes. Also, the pressure fluctuation for fully developed turbulent flow in curved pipes is relatively damped.

\subsubsection{Effect of DRAs on flow transition and critical Reynolds number}

The transition from laminar to turbulent flow regime in curved pipes is gradual and sometimes difficult to identify. This transition is even more gradual in the case of non-Newtonian drag-reducing fluid flow in curved pipes [33], [88], [111]. A delayed and gradual transition from laminar to turbulent regime occurs for flow of DRAs through curved pipes [23]. Two factors could be responsible for this: turbulence suppression in curved flow geometry, and effect of drag-reducing agent on flow transition. Effect of DRAs on flow transition in curves and bends depends on the curvature of the bend and concentration of drag-reducing agent. Fig. 5 shows that the critical Reynolds number decreases with curvature and increases with concentration of surfactant. Transition to turbulent flow occurred when the wall shear of the DRA exceeded the critical wall shear stress under strong mechanical load at high Reynolds numbers.

The critical Reynolds numbers also depend on the temperature especially in the turbulent regime. In separate experiments conducted by Inaba et al. [70] and Aly et al. [22] using surfactants in the temperature range of 5 $-20^{\circ} \mathrm{C}$, it was observed that critical modified Reynolds number $N_{\mathrm{Re}_{\text {crit }}^{\prime}}$ increases with increase in temperature. This is associated with the critical wall shear stress at the wall which increases with temperature.

\subsection{Friction factor correlations for single phase flow in curved pipes}

Several theoretical and empirical models are available for predicting friction factor of non-Newtonian fluids through curved pipes. In majority of the correlations, friction factors are simple functions of the Dean number and curvature ratio, $\frac{a}{R}$, of the pipe. In general, at low Dean number the friction factor can be defined as a sole function of Dean number, $N_{\text {Dn }}$. At higher Dean numbers, the frictional characteristic of flow not only depend on $N_{\mathrm{Dn}}$, but also on $\frac{a}{R}$. Most of these correlations appear in the form of ratios of friction factors in curved pipes to that in straight pipes at the same conditions. Some researchers [86], [112], [113] presented friction factor correlation for drag-reducing fluids in both straight and curved pipes in terms of the Deborah number $N_{\text {De }}$ defined as:

$N_{\text {De }}=\frac{\text { characteristic fluid time }}{\text { characteristic flow time }}(16)$

Table 1 presents a summary of friction factor correlation for non-Newtonian fluids in curved pipes.

Table 1. Friction factor correlations for non-Newtonian fluids in curved pipes

\begin{tabular}{|c|c|c|c|}
\hline Author//DRA type & Test fluid & Correlation & Remarks \\
\hline$[86]$ & Polyacrylamide & $\frac{f_{c}}{f_{c, o}}=0.2+\frac{0.8}{1+N_{D e^{\prime}}^{0.8}}$ & $\begin{array}{l}N_{D e^{\prime}}=\frac{w}{w_{0.6}} \text { Where } w_{0.6} \\
\text { are the values of } w \text { at } \\
\frac{f_{c}}{f_{c, o}}=0.6\end{array}$ \\
\hline$[114]$ & $\begin{array}{l}\text { Polyacrylamide } \\
\text { Polyethylene oxide } \\
\text { Carboxymethyl cellulose }\end{array}$ & $\begin{array}{l}f_{\mathrm{CL}}= \\
\left(9.069-9.438 n+4.37 n^{2}\right)\end{array}$ & 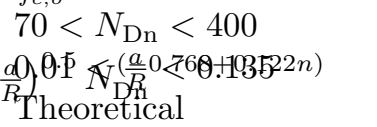 \\
\hline
\end{tabular}




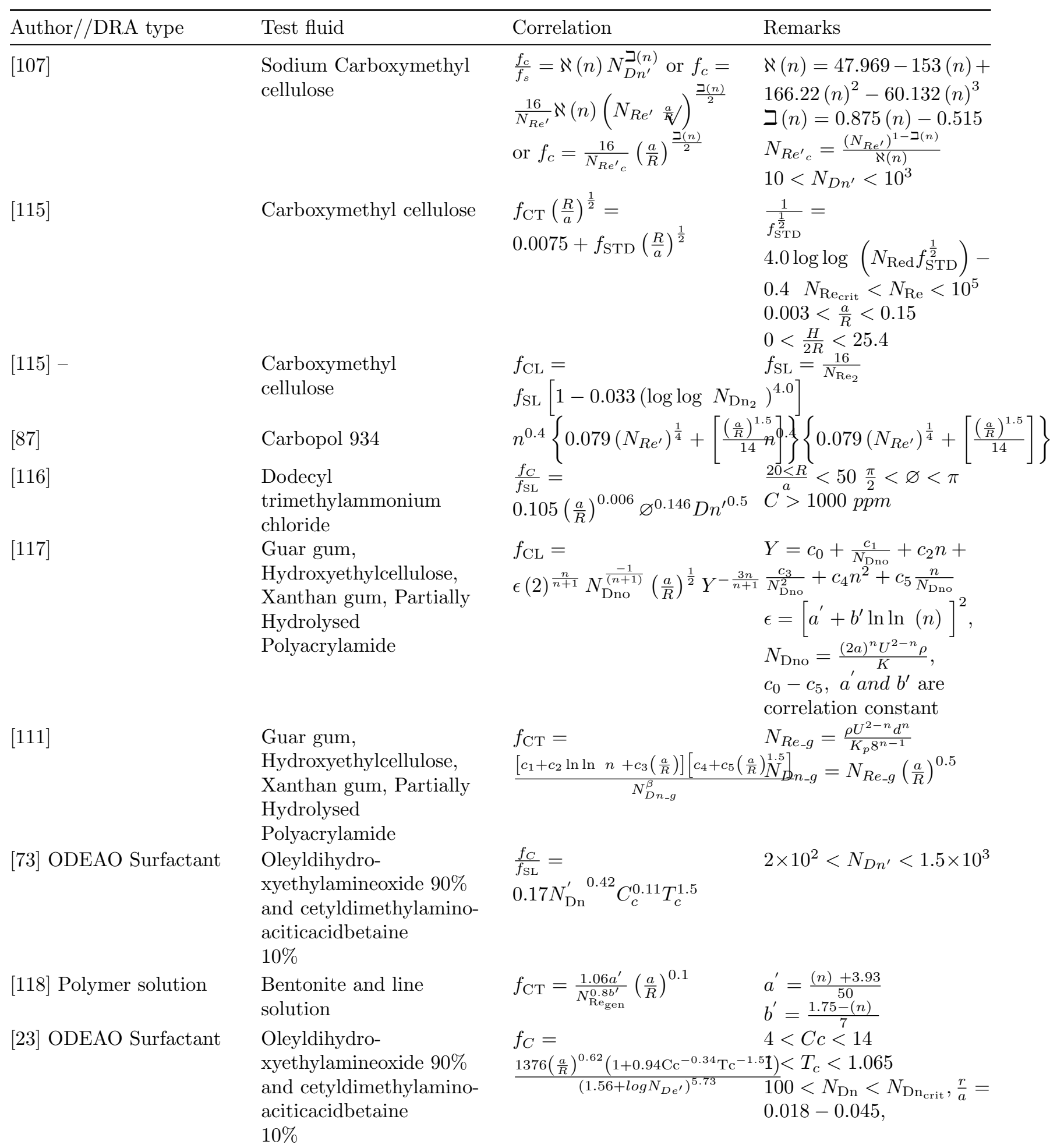




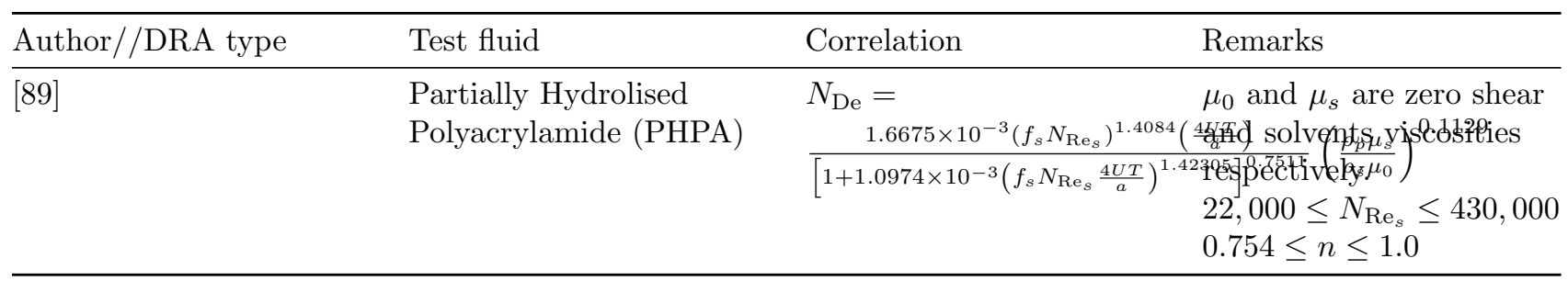

Table 2. Flux models for flow on non-Newtonian fluids in curved pipes.

\begin{tabular}{|c|c|c|}
\hline Author & Correlation & Remarks \\
\hline [98] & $\begin{array}{l}\frac{Q_{c}}{Q_{s}}= \\
1-\left(\frac{1}{576} L\right)^{2}(0.03058-0.06426 m)\end{array}$ & $m=0.5 \alpha_{3}^{1} Q_{s}=\frac{W_{0} \pi a^{2}}{2}$ Low $N_{\mathrm{Dn}}$ \\
\hline [83] & $\begin{array}{l}\frac{Q_{c}}{Q_{s}}=1- \\
\left(\frac{L}{576}\right)^{2}\left(0.0306+0.4607 \alpha_{2}^{\prime}+0.1982 \alpha_{3}^{\prime}\right.\end{array}$ & $\begin{array}{l}\frac{Q_{c}}{Q_{s}}=1- \\
c_{3}\left(\frac{L}{576}\right) 1^{2} 2\left(80 x^{\prime} 3 \theta 62570836 \alpha^{\prime}\right) \alpha_{2}^{\prime}+0.1982 \alpha_{3}^{\prime}+112.8 \alpha_{5}^{\prime}\end{array}$ \\
\hline$[100]$ & $\begin{array}{l}\frac{Q_{s}}{Q_{c}}=1+(N-1) 6.3 \times \\
10^{-4} \frac{R}{a}\left[0.14\left(\frac{R}{a}\right)^{0.5}+4.5\right]^{2}\left(\frac{Q_{c_{\text {Newtonid }}}}{Q_{s}}\right.\end{array}$ & $\begin{array}{l}N \text { is the number of bends or } \\
\text { cirurve } \frac{Q_{c_{\text {Newtonian }}}}{Q_{s}} \text { is taken from } \\
\text { the master curve } 6.3 \times 10^{-4} \text { is } \\
\text { adjustable parameter that is } \\
\text { dependent on geometry }\end{array}$ \\
\hline$[90]$ & $\begin{array}{l}\frac{Q_{s}}{Q_{c}}=1+ \\
\frac{1}{48}\left(\frac{a}{R}\right)^{2}\left[1-N_{\operatorname{Re}}^{2}\left(\frac{11}{360}+N_{\operatorname{Re}}^{2} \frac{1541}{87091200}\right)\right.\end{array}$ & 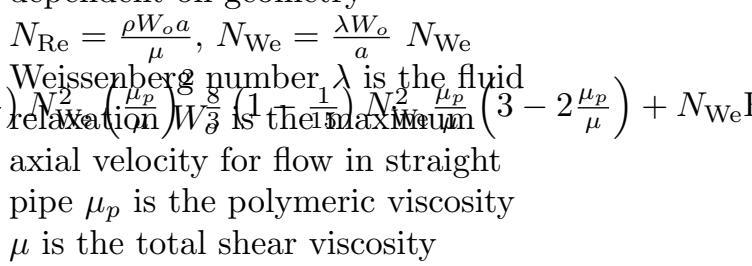 \\
\hline
\end{tabular}

\subsection{Flux models for non-Newtonian fluids}

Several theoretical and semi-theoretical solutions to the governing equations of flow in curved pipes have resulted in series equations for fluid flux. Most of the theoretical correlations proposed for fluid flux in curved pipes are functions of material constants $\left(\alpha_{1}, \alpha_{2}, \alpha_{3}, \alpha_{5}, \beta_{1}\right.$ and $\left.\beta_{2}\right)$. The normal stress difference for flow in curved pipes involve $\alpha_{2}$ and $\alpha_{3}$ only and the term $\left(\alpha_{5}+\beta_{1}\right)$ represents the departure from a constant viscosity. For straight pipe flow the flux is determined by the viscosity constant $\alpha_{1}, \alpha_{5}$ and $\beta_{1}$ and is independent of normal stress terms $\alpha_{2}$ and $\alpha_{3}$ [63]. The material constants are also expressed as, $\alpha_{2}^{\prime}=$ $\frac{\alpha_{2}}{\left(\rho a^{2}\right)}, \alpha_{3}^{\prime}=\frac{\alpha_{3}}{\left(\rho a^{2}\right)}, \alpha_{5}^{\prime}=\frac{\alpha_{5} \alpha_{1}}{\left(\rho^{2} a^{4}\right)}$ and $\beta_{1}^{\prime}=\frac{\beta_{1} \alpha_{1}}{\left(\rho^{2} a^{4}\right)}$. Others presented their correlations for flow rates as functions of Weissenberg number, We, Reynolds number and ratio of polymeric to shear viscosity. Table 2 gives a summary of fluid flux models.

\subsection{Two phase liquid-liquid flow and the effect of drag-reducing agents on two- phase (gas-liquid and liquid-liquid) flow in curved pipes}

Limited reports are available in open literature on the effect of drag-reducing agents on gas-liquid flow in bends and curves. In the knowledge of the Authors, no report is available in the public domain that investigates the effect of drag-reducing agents on liquid-liquid flows in bends and curves. Unlike like the flow of gas-liquids in curves and bends, two-phase liquid-liquid flows in curves and bends have received little attention till date. Research has shown that liquid-liquid properties such as density, viscosity and interfacial 
tension have profound effects of pressure drop and flow pattern characteristics [7], [119], [120]. It has also been establish that flow patterns and fluid characteristics such as interfacial tension play important role in determining the effectiveness of drag reducing agents [121], [122].

\subsubsection{Effect of drag-reducing agents on gas-liquid flows in curved pipes}

Though there is limited literature on the flow on gas-non-Newtonian fluids in curved pipes, a reasonable body of knowledge exist for the case of Gas-Newtonian fluid flows in curved pipes. A few reports on the flow of air-CMC solution in helical coils have shown that the CMC solution has a significant influence on both in-situ volume fraction and pressure drop [123]. Pressure drop reduction was reported by Mujawar and Rao [120] when the drag coefficient approach [124] was used for analysis but not when the Lockhart and Martinelli [122] approach was used. This highlights the limitations to the applicability of these correlations for predicting gas-non-Newtonian liquid flows. Some more recent reports on air-SCMC systems have shown that polymer concentration, pipe curvature, pipe diameter and to a lesser extent helical coil pitch does influence frictional pressure losses, phase distribution and liquid holdup of gas-liquid flows in helical coils [126]-[128]. Although the objective of these studies was not to determine DR, useful information on DR can be derived from them. It is important to state here that SCMC solutions can behave as pseudo-plastic, dilatant or thixotropic fluids depending on the concentration and temperature of the polymer solution [129]. In general, however, it behaves as a shear thinning (pseudo-plastic) fluid at low concentrations ( $<1 \mathrm{wt} \%)$. This behaviour probably explains the results of Biswas and Das [123] where an increase in frictional pressure loss with increase in fluid viscosity (increase in concentration) was reported. The implication of the result is that DR (if any) occurs at low polymer concentration. Thandlam et al. [124] reported that stratified flow (ST) regime (in the case of air-SCMC solution) occupied a larger region of the flow pattern map compared to air-water flow. The extension of the ST flow regime is an indication of turbulence suppression and this effect has been reported for DRPs application in straight pipes [122]. In a separate report that focuses on mass transfer characteristics of air-SCMC solution in helical coils, it was reported that mass transfer $\left(\mathrm{k}_{\mathrm{l}}\right)$ was higher for Newtonian water flow than for SCMC solution and it decreases with SCMC concentration [128]. Since mass transfer is proportional to frictional force [130], [131], it may be inferred that the frictional force in water was higher than that of SCMC at the test concentration of $<3 \mathrm{~kg} / \mathrm{m}^{3}$ used in that study. The effective viscosity for shear thinning liquids is higher when it flows as a single phase liquid in coils than when it flows together with gas [132]. This is due to the higher shear rates for two-phase gas liquid flows compared to the simple shearing flow of single-phase liquid flow. The implication of this is higher drag reduction than single phase flow of polymer solution due to reduced effective viscosity. It may be deduced that for shear thickening liquids lower drag reduction would be obtainable in two-phase flow compared to single phase flow (though no research is available to confirm this postulation). Clearly further research is needed to interrogate the effect of drag-reducing agents on gas-liquids flows in curved pipes.

\subsection{Knowledge gap}

The review revealed key findings that go a long way in answering questions regarding the effects of dragreducing polymers, surfactants and micro-bubbles for flows in curved pipes. However, certain research gaps were identified particularly in the area of two-phase liquid-liquid drag reduction in curved pipe flows.

\subsection{Single phase flow in curved pipes}

Several studies have been carried out to investigate the non-Newtonian effects of drag-reducing agents in single phase liquid flow in curved pipes. However, some research gaps remain. Some of the areas where further works are needed are outlined below:

1. Further studies are required to investigate the effect of drag-reducing agents on secondary flows. Understanding of the mechanism of interaction of polymer and surfactant macromolecules with secondary 
flow streamlines could provide answers to the reported trends in the laminar flow regime.

2. For flow in bends (the flow is not hydrodynamically developed) the effect of drag-reducing agents on flow separation and reattachment is not yet understood. The reduced drag reduction in bends (compared to coiled pipes) has been linked to flow separation and reattachment. Thus, insight into the effect of DRAs on flow separation and reattachment could provide answers to this observation.

3. Most of the existing researches on drag reduction in curved pipes are centred on coils. Limited studies have been carried out to interrogate the effect of drag-reducing agents for flows in bends. In particular, research is needed to investigate the effect of bend angles on drag reduction.

4. Detailed study of the effect of pipe diameter on the effectiveness of polymer, surfactant and microbubble drag reduction is required.

5. The effect of micro-bubble size on drag reduction for curved pipe flows is another area where research is required. The exiting studies are limited to straight channel flow and reports on the effect of bubble size on drag reduction are conflicting.

6. Proper understanding of mechanism of polymer and surfactant drag reduction in curved pipes could provide a means of quantitatively linking polymer and surfactant properties to the reported drag reduction.

7. The maximum drag reduction for polymer and surfactant DRAs in curved pipes is an area that needs to be explored further. There is the need to further investigate the effects of pipe geometry such as diameter and curvature on DRAs in curved pipes.

8. The synergistic effect of polymer-surfactant combination may also be explored.

9. The effects of temperature, dissolved salts and silt on drag reduction by additives in pipe bends and curves needs to be further explored. Reports on these are either scanty or conflicting.

10. Research into the effect of drag-reducing agents on velocity profile distribution in curved pipes is not available in open literature. Studies in this area could provide more explanation to this behaviour observed in straight pipe flow.

\subsection{Two-phase flows in curved pipes}

Studies into the effects of drag-reducing agents on liquid-liquid flows in curved pipes are lacking and those pertaining to gas-liquid flow are scanty. Experience from single phase flow shows that straight pipe data cannot be extended to account for observations in curved pipes. Some of the areas where there are need for further research include the following.

1. Effects of DRAs on phase distribution, pressure drop among others for two-phase liquid-liquid flows through curved pipes is yet to receive proper scholarly attention.

2. The effect of pipe orientation on pressure drop, phase distribution and effectiveness of drag-reducing agents for two-phase flows in curved pipes needs to be investigated.

3. There is limited study that investigates the effects of temperature, dissolved salts and silt on drag reduction for liquid-liquid flows in curved pipes. Research in this area could provide practical solutions for field scale operations.

4. Researches in the area of drag reduction in two-phase flows in bends are lacking. The few existing literatures have focused on coiled pipe flows. Insight into the effect of drag-reducing agent on phase distribution in pipe bends could go long way in improving process management and safety.

\subsection{Conclusion}

The importance of pipe fittings (e.g. bends) and curves in pipeline transport has necessitated the need for more understanding of the hydrodynamics of flow in them. Core among the areas of research interest is drag reduction by additives for single- and two-phase flows in curved pipes. It was shown in this review that significant drag reduction in curved pipes can be achieved using polymer, surfactant and micro-bubbles. However, the drag reductions reported in curved pipes are generally lower than the corresponding drag 
reduction in straight pipes. In general drag reduction decreased with increase in curvature due to higher centrifugal forces. Similar to straight channel flows, drag reduction by additives is predominantly in the turbulent flow regime, but a few studies reported DR in the laminar flow regime. Beyond certain critical Reynolds number, in the turbulent regime, drag reduction by additives reduced with increase in Reynolds number but prior to this the reverse is the case. This has been attributed to uneven distribution of air bubbles or degradation of polymer or surfactant as the case may be. It was also seen that the effectiveness of polymer and surfactant DRAs increased with concentration until a threshold concentration is attained. Likewise, the effectiveness of micro-bubble as drag-reducing agent in curved channel flow generally increased with air fraction. It was also reported that drag reduction depends on other factors such as temperature and the presence of dissolved salts. This review examined correlations for maximum drag reduction asymptote for flow of polymer and surfactants in curved pipes. It was seen that the maximum drag reduction asymptote differed between curved and straight pipes and between polymers and surfactants. Single phase drag reduction correlations for flows in curves of polymers and surfactants were presented in this review. A brief review of drag reduction for two-phase gas-liquid flows in coils was also provided. Though drag reduction was reported in the limited research in this area, no definite conclusion could be drawn on the effect of dragreducing agents on gas-liquid flows in curved pipes. With the prospect of reduced pumping cost, improved operational flexibility and transport safety, research is expected to continue in this area in the future. Extension of hydrodynamic and drag reduction studies to three-phase gas-liquid-liquid flows is also essential owing to the occurrence of this flow in petroleum installations.

\section{CONFLI CT OF INTEREST}

The Authors wish to state that there are no known conflict of interest arising from the publication of this manuscript

\section{Nomenclature}

$\mathrm{R}=$ Coil radius or Radius of curvature of curve

$\mathrm{a}=$ pipe diameter

$\mathrm{a} / \mathrm{R}=$ curvature ratio

$\mathrm{N}_{\mathrm{Dn}}=$ Dean number

$\mathrm{N}_{\mathrm{Re}}=$ Reynolds number

$\mathrm{N}_{\mathrm{Re}}$, = Modified Reynolds number

$\mathrm{N}_{\mathrm{we}}=$ Weissenberg number

$\mathrm{N}_{\text {Re_crit }}=$ Critical Reynolds number for flow transition from laminar to turbulent flow.

$\mathrm{H}=$ Coil pitch

$\mathrm{L}=$ original definition of Dean number

$\mathrm{f}=$ friction factor

$\mathrm{K}=$ consistency factor of power law model

$K_{p}=$ consistency factor from pipe viscometer, $\frac{\mathrm{Ib}_{f} s^{n}}{\mathrm{ft}^{2}}$

$\mathrm{n}=$ behavioural index (exponent of power law fluids)

$\mathrm{S}=$ Slip ratio

$\mathrm{P}=$ Static pressure head

$\mathrm{T}=$ fluid relaxation time

$\beta=$ inlet water fraction 
$C c=$ dimensionless concentration defined as the ratio of surfactant concentration to the lower critical concentration in a straight pipe of $C=250 \mathrm{ppm}$ for ODEAO surfactant at which the rod-like micelles cannot be formed and there is no drag reduction effect.

$T c=$ dimensionless temperature, defined as the ratio of actual surfactant solution to the lower critical absolute temperature of $275 \mathrm{~K}$ where there is no drag reduction effect.

$\varnothing$ is the angle of curvature measured from the inlet of the curved tube.

$m=\frac{1}{2} \alpha_{3}^{\prime}$, a measure of the elasticity of the fluid

$r_{1}=\frac{r}{a}$

$\Psi=$ secondary flow stream function

$h_{r}=$ pipe roughness projections

Subscripts

$\mathrm{s}=$ solvent

$\mathrm{p}=$ polymer

$\mathrm{w}=$ water

\section{References}

[1] M. Abdulkadir, D. Zhao, S. Sharaf, L. Abdulkareem, I. S. Lowndes, and B. J. Azzopardi, "Interrogating the effect of 90 o bends on air-silicone oil flows using advanced instrumentation," Chem. Eng. Sci. , vol. 66, no. 11, pp. 2453-2467, 2011.

[2] B. S. K. Ahmed, "A study of gas lift on oil/water flow in vertical risers," Cranfield University, 2014.

[3] P. Onubi, L. C. Edomwonyi-Otu, and N. Yusuf, "Experimental and neural network modelling of polymer drag reduction in 180 bends," Results Mater. , vol. 1, no. August, p. 100012, 2019.

[4] L. C. Edomwonyi-Otu, M. Chinaud, and P. Angeli, "Effect of drag reducing polymer on horizontal liquid-liquid flows," Exp. Therm. Fluid Sci. , vol. 64, pp. 164-174, 2015.

[5] L. C. Edomwonyi-Otu and D. O. Adelakun, "Effect of heavy molecular weight polymer on quality of drinking water," Mater. Today Commun., vol. 15, pp. 337-343, 2018.

[6] B. A. Tom, "Some observations on the flow of linear polymer solutions through straight tubes at large Reynolds numbers," inFirst International Congress on Rheology , 1948, vol. 2, pp. 135-141.

[7] N. Yusuf, Y. Al-Wahaibi, T. Al-Wahaibi, A. Al-Ajmi, A. S. Olawale, and I. A. Mohammed, "Effect of oil viscosity on the flow structure and pressure gradient in horizontal oil - water flow," Chem. Eng. Res. Des. , vol. 90, no. 8, pp. 1019-1030, 2012.

[8] A. Abubakar, T. Al-Wahaibi, A. R. Al-Hashmi, Y. Al-Wahaibi, A. Al-Ajmi, and M. Eshrati, "Influence of drag-reducing polymer on flow patterns, drag reduction and slip velocity ratio of oil-water flow in horizontal pipe," Int. J. Multiph. Flow, vol. 73, pp. 1-10, 2015.

[9] K. B. Fox, M. S. Bainum, and C. Chemical, "A new synthetic polymer provides improved drag reduction in coiled-tubing operations in North Louisiana," in SPE /ICoTA Coiled Tubing E W Well Intervention Conference (the Woodlands, Tx, 3/23-24/2010) Proceedings, 2010, no. 201029, pp. 1-8.

[10] K. Gasljevic and E. F. Matthys, "Friction and heat transfer in drag-reducing surfactant solution flow through curved pipes and elbows," Eur. J. Mech. / B Fluids, vol. 28, no. 5, pp. 641-650, 2009.

[11] A. H. A. Kamel, "Drag reduction behavior of polymers in straight and coiled tubing at elevated temperature," Electron. Sci. J. Oil Gas Bus., vol. 1, no. 1, pp. 107-128, 2011. 
[12] J. Chen, C. Tsai, Y. Kehr, L. Horng, K. Chang, and L. Kuo, "An experimental study of drag reduction in a pipe with superhydrophobic coating at moderate Reynolds numbers," in EPJ Web of Conferenece 6 , 2010, vol. 19005, pp. 1-8.

[13] A. Abubakar, T. Al-Wahaibi, Y. Al-Wahaibi, A. R. Al-Hashmi, and A. Al-Ajmi, "Roles of drag reducing polymers in single- and multi-phase flows," Chem. Eng. Res. Des., vol. 92, no. 11, pp. 2153-2181, Nov. 2014 .

[14] H. A. Abdulbari, A. Shabirin, and H. N. Abdurrahman, "Bio-polymers for improving liquid flow in pipelines-A review and future work opportunities," J. Ind. Eng. Chem. , vol. 20, no. 4, pp. 1157-1170, Jul. 2014.

[15] J. G. Savins, "Drag reduction characteristics of solutions of macromolecules in turbulent pipe flow," SPEJ , vol. 3, no. 4, pp. 203-214, 1964.

[16] M. Kostic, "On turbulent drag and heat transfer phenomena reduction and laminar heat transfer enhancement in non-circular duct flow of certain non-Newtonian fluids," Int. J. Heat Mass Transf. , vol. 37, pp. 133-147, 1994.

[17] G. Aguilar, K. Gasljevic, and E. F. Matthys, "Coupling between heat and momentum transfer mechanisms for drag-reducing polymer and surfactant solutions," ASME J. Heat Transf. , vol. 121, pp. 796-802, 1999.

[18] K. Gasljevic and E. F. Matthys, "Improved quantification of the drag reduction phenomenon through turbulence reduction parameters," J. Nonnewton. Fluid Mech. , vol. 84, no. 2-3, pp. 123-130, 1999.

[19] Y. Zhou, "Theoritical and experimental studies of power-law fluid flow in coiled tubing," University of Oklahoma, 2006.

[20] M. S. N. Kazi, G. G. Duffy, and X. D. Chen, "Heat transfer in the drag reducing regime of wood pulp fibre suspensions," Chem. Eng. J., vol. 73, no. 3, pp. 247-253, 1999.

[21] G. Aguilar, K. Gasljevic, and E. F. Matthys, "Reduction of friction in fluid transport : experimental investigation," Rev. Mex. Fis., vol. 52, no. 5, pp. 444-452, 2006.

[22] X. Zhang, L. Liu, L. Cheng, Q. Guo, and N. Zhang, "Experimental study on heat transfer and pressure drop characteristics of air - water two-phase flow with the effect of polyacrylamide additive in a horizontal circular tube," Int. J. Heat Mass Transf. , vol. 58, no. 1-2, pp. 427-440, 2013.

[23] W. I. A. Aly, H. Inaba, N. Haruki, and A. Horibe, "Drag and heat transfer reduction phenomena of drag-reducing surfactant solutions in straight and helical pipes," Trans. ASME, vol. 128, no. August 2006, pp. 800-810, 2006.

[24] M. D. Warholic, D. K. Heist, M. Katcher, and T. J. Hanratty, "A study with particle-image velocimetry of the influence of drag-reducing polymers on the structure of turbulence," Exp. Fluids, vol. 31, no. 5, pp. 474-483, Nov. 2001.

[25] T. Al-Wahaibi, M. Smith, and P. Angeli, "Effect of drag-reducing polymers on horizontal oil - water flows," J. Pet. Sci. Eng., vol. 57, pp. 334-346, 2007.

[26] M. Al-Yaari, A. Soleimani, B. Abu-Sharkh, U. Al-Mubaiyedh, and A. Al-Sarkhi, "Effect of drag reducing polymers on oil - water flow in a horizontal pipe," Int. J. Heat Fluid Flow, vol. 35, no. June 2009, pp. 516-524, 2009.

[27] D. K. Pragya, R. Naidu, T. Mandal, K. Majumda, and S. Kumar, "Flow pattern shifting and drag reduction in oil-water flow pipe," IMPACT Int. J. Res. Eng. Technol., vol. 2, no. 2, pp. 245-252, 2014.

[28] A. M. Fsadni, J. P. M. Whitty, and M. A. Stables, "A brief review on frictional pressure drop reduction studies for laminar and turbulent flow in helically coiled tubes," Appl. Therm. Eng. , vol. 16, pp. 1-24, 
2016.

[29] P. S. Virk, "Drag Reduction Fundamentals," AlChE J., vol. 21, no. 4, pp. 625-656, 1975.

[30] C. White and M. G. Mungal, "Mechanics and Prediction of Turbulent Drag Reduction with Polymer Additives," Annu. Rev. Fluid Mech., vol. 40, no. 1, pp. 235-256, Jan. 2008.

[31] S. N. Shah and Y. Zhou, "Maximum drag reduction asymptote of polymeric fluid flow in coiled tubing," J. Fluids Eng., vol. 131, no. January 2009, pp. 1-9, 2009.

[32] W. J. Han, Y. Z. Dong, and H. J. Choi, "Applications of Water-Soluble Polymers in Turbulent Drag Reduction," Process, vol. 5, no. 24, pp. 1-27, 2017.

[33] S. N. Shah and Y. Zhou, "An experimental study of drag reduction of polymer solutions in coiled tubing," Soc. Pet. Eng., vol. SPE 68416, pp. 1-14, 2001.

[34] Y. Zhou, S. N. Shah, and P. V Gujar, "Effects of coiled-tubing curvature on drag reduction of polymeric fluids," SPE Prod. Oper. , vol. 21, no. 1, pp. 134-141, 2004.

[35] P. S. Srinivasan, S. S. Nandapurkar, and F. A. Holland, "Friction factors for coils," Trans. Instn. Chem. Engr., vol. 48, pp. T156-T161, 1970.

[36] S. Liu and J. H. Masliyah, "Axially invariant laminar flow in helical pipes with a finite pitch," J. Fluid Mech. , vol. 251, pp. 315-353, 1993.

[37] J. L. Zakin, J. Myska, and Z. Chara, "New limiting drag reduction and velocity profile asymptotes for nonpolymeric additives systems," AIChE J. , vol. 42, no. 12, pp. 3544-3546, 1996.

[38] A. H. Kamel and S. N. Shah, "Maximum drag reduction asymptote for surfactant-based fluids in circular coiled tubing," J. Fluids Eng. , vol. 135, no. 3, p. 031201, 2013.

[39] L. C. Edomwonyi-Otu, M. Chinaud, and P. Angeli, "Effect of drag reducing polymer on horizontal liquid-liquid flows," Exp. Therm. Fluid Sci. , vol. 64, pp. 164-174, 2015.

[40] T. Al-Wahaibi et al. , "Experimental study on the effect of drag reducing polymer on flow patterns and drag reduction in a horizontal oil-water flow," Int. J. Heat Fluid Flow, vol. 37, pp. 74-80, Oct. 2012.

[41] L. C. Edomwonyi-Otu, "Drag Reduction in Oil-Water Flows," University College London, UK, 2015.

[42] B. A. Jubran, Y. H. Zurigat, and M. F. A. Goosen, "Drag reducing agents in multiphase flow pipelines : Recent trends and future needs drag reducing agents in multiphase flow pipelines : Recent trends and future needs," Pet. Sci. Technol., vol. 23, no. February, pp. 1403-1424, 2005.

[43] S. M. Hosseini-Nasab, M. Taal, P. L. J. Zitha, and M. Sharifi, "Effect of Newtonian and non-Newtonian viscosifying agents on stability of foams in enhanced oil recovery. Part I: under bulk condition," Iran. Polym. J. , vol. 28, no. 4, pp. 291-299, 2019.

[44] Z. Y. Gui, H. R. Wang, Y. Gao, C. Lu, and S. J. Cheng, "Morphology and melt rheology of biodegradable poly(lactic acid)/poly(butylene succinate adipate) blends: Effect of blend compositions," Iran. Polym. J. (English Ed. , vol. 21, no. 2, pp. 81-89, 2012.

[45] R. Pourshooshtar, Z. Ahmadi, and F. A. Taromi, "Formation of 3D networks in polylactic acid by adjusting the cross-linking agent content with respect to processing variables: a simple approach," Iran. Polym. J. (English Ed. , vol. 27, no. 5, pp. 329-337, 2018.

[46] C. Zareie, M. V. Sefti, A. R. Bahramian, and M. B. Salehi, "A polyacrylamide hydrogel for application at high temperature and salinity tolerance in temporary well plugging," Iran. Polym. J. (English Ed., vol. 27, no. 8, pp. 577-587, 2018.

[47] J. L. Lumley, "Drag Reduction in Turbulent Flow by Polymer Additives," J. Polym. Sci. Macromol. Rev. , vol. 7, no. 1, pp. 263-290, 1973. 
[48] T. Min, J. Y. Yoo, H. Choi, and D. D. Joseph, "Drag reduction by polymer additives in a turbulent channel flow," J. Fluid Mech. , vol. 486, pp. 213-238, 2003.

[49] I. Sher and G. Hetsroni, "A mechanistic model of turbulent drag reduction by additives," Chem. Eng. Sci. , vol. 63, no. 7, pp. 1771-1778, Apr. 2008.

[50] R. Armstrong and M. S. Jhon, "Turbulence induced change in the conformation of polymer molecules," J. Chem. Phys., vol. 79, pp. 3143-3147, 1983.

[51] A. V. Shenoy, "A review on drag reduction with special reference to micellar systems," Colloid Polym. Sci. , vol. 262, pp. 319-337, 1984.

[52] L. E. Wedgewood, D. N. Ostrov, and B. R. Bird, "A finitely extensible beadspring chain model for dilute polymer solutions," J. Non-Newton. Fluid Mech. , vol. 40, pp. 119-139, 1991.

[53] F. C. Li, W. H. Cai, H. N. Zhang, and Y. Wang, "Inuence of polymer additives on turbulent energy cascading in forced homogeneous isotropic turbulence studied by direct numerical simulations," Chin. Phys. $B$, vol. 21, p. 114701, 2012.

[54] V. E. Terrapon, Y. Dubief, P. Moin, E. S. G. Shaqfeh, and S. K. Lele, "Simulated polymer stretch in a turbulent flow using Bronian dynamics," J. Fluid Mech., vol. 504, pp. 61-71, 2004.

[55] Y. Dubief, C. M. White, V. E. Terrapon, E. S. G. Shaqfeh, P. Moin, and S. K. Lele, "On the coherent drag-reducing and turbulence-enhancing behavior of polymers in wall flows," J. Fluid Mech. , vol. 514, pp. 271-280, 2004.

[56] Y. Dubief, V. E. Terrapon, C. M. White, E. S. G. Shaqfeh, P. Moin, and S. K. Lele, "New answers on the ineraction between polymers and vortices in turbulent flows," Flow, Turbul. Combust. , vol. 74, pp. $311-329,2005$.

[57] C. D. Dimitropoulos, Y. Dubief, E. S. G. Shaqfeh, and P. Moin, "Direct numerical simulation of polymerinduced drag reduction in turbulent boundary layer flow of inhomogeneous polymer solutions," J. Fluid Mech. , vol. 566, pp. 153-162, 2006.

[58] J. M. J. Den Toonder, M. A. Hulsen, G. D. C. Kuiken, and F. T. M. Nieuwstadt, "Drag reduction by polymer additives in a turbulent pipe flow: numerical and laboratory experiments," J. Fluid Mech., vol. 337, pp. 193-231, Apr. 1997.

[59] M. D. Warholic, H. Massah, and T. J. Hanratty, "Influence of drag-reducing polymers on turbulence: effects of Reynolds number, concentration and mixing," Exp. Fluids, vol. 27, no. 5, pp. 461-472, Oct. 1999.

[60] M. Itoh, S. Tamano, K. Yokota, and M. Ninagawa, "Velocity measurement in turbulent boundary layer of drag-reducing surfactant solution," Phys. Fluids, vol. 17, p. 075107, 2005.

[61] I. Zadrazil, A. Bismarck, G. F. Hewitt, and C. N. Markides, "Shear layers in the turbulent pipe flow of drag reducing polymer solutions," Chem. Eng. Sci., vol. 72, pp. 142-154, Apr. 2012.

[62] L. C. Edomwonyi-Otu and P. Angeli, "Separated oil-water flows with drag reducing polymers," Exp. Therm. Fluid Sci., vol. 102, no. September 2017, pp. 467-478, 2019.

[63] H. A. Barnes and K. Walters, "On the flow of viscous and elastico-viscous liquids through straight and curved pipes," Proc. Roy. Soc. Lond. A, vol. 314, no. 1516, pp. 85-109, 1969.

[64] P. E. Rouse, "A theory of the linear viscoelastic properties of dilute solutions of coiling polymers," J. Chem. Phys., vol. 21, no. 1953, pp. 1272-1280, 1953.

[65] H. Y. Tsang and D. F. James, "Reduction of secondary motion in curved tubes by polymer additives reduction of secondary motion in curved tubes by polymer additives," J. Rheol. (N. Y. N. Y)., vol. 24, no. 5, pp. 589-601, 1980. 
[66] D. F. James and J. H. Saringer, "Extensional flow of dilute polymer solutions," J. Fluid Mech. , vol. 97, no. 4, pp. 655-671, 1980.

[67] K. Gasljevic and E. F. Matthys, "On saving pumping power in hydronic thermal distribution systems through the use of drag-reducing additives," Energy Build. , vol. 20, no. 1, pp. 45-56, 1993.

[68] Y. Kawaguchi, T. Segawa, Z. Feng, and P. Li, "Experimental study on drag-reducing channel flow with surfactant additives-Spatial structure of turbulence investigated by PIV system," Int. J. Heat Fluid Flow , vol. 23, no. 5, pp. 700-709, 2002.

[69] T. Saeki, "Flow properties and heat transfer of drag-reducing surfactant solutions," Developments in Heat Transfer. InTech, pp. 331-349, 2011.

[70] H. Bewersdorff and D. Ohlendon, "The behaviour of drag-reducing cationic surfactant solutions," Colloid Polym. Sci. , vol. 266, pp. 941-953, 1988.

[71] G. D. Rose and K. L. Foster, "Drag reduction and rheological properties of cationic viscoelastic surfactant formulations," J. Nonnewton. Fluid Mech., vol. 31, no. 1, pp. 59-85, 1989.

[72] B. Lu, X. Li, Y. Talmon, and J. L. Zakin, "A non-viscoelastic drag reducing cationic surfactant system," J. Non-Newtonian Fluid Mech. , vol. 71, pp. 59-72, 1997.

[73] H. Inaba, W. I. A. Aly, N. Haruki, and A. Horibe, "Flow and heat transfer characteristics of drag reducing surfactant solution in a helically coiled pipe," Heat Mass Transf. , vol. 41, pp. 940-952, 2005.

[74] J. Myska and V. Mik, "Degradation of surfactant solutions by age and by a flow singularity," Chem. Eng. Process. Process Intensif. , vol. 43, no. 12, pp. 1495-1501, 2004.

[75] T. P. Elson and J. Garside, "Drag reduction in aqueous cationic soap solutions," J. Nonnewton. Fluid Mech. , vol. 12, no. 2, pp. 121-133, 1983.

[76] B. van der Plas and M. Golombok, "Reservoir resilience of viscoelastic surfactants," J. Pet. Explor. Prod. Technol. , vol. 7, no. 3, pp. 873-879, 2017.

[77] Y. Hassan and J. Ortiz-Villafuerte, "Experimental study of micro-bubble drag reduction using particle image velocimetry," 11th Int. Symp. . . , no. 1999, pp. 1-11, 2002.

[78] M. M. E. Shatat, S. Yanase, T. Takami, and T. Hyakutake, "Drag reduction effects of micro-bubbles in straight and helical Pipes," J. Fluid Sci. Technol. , vol. 4, no. 1, pp. 156-167, 2009.

[79] M. E. McCormick and R. Bhattacharyya, "Drag reduction of a submersible hull by electrolyis," Nav. Eng. J. , pp. 11-16, 1973.

[80] N. K. Madavan, S. Deutsch, and C. L. Merkle, "Numerical investigation into the mechanisms of microbubble drag reduction," J. Fluids Eng. , vol. 127, pp. 370-377, 1985.

[81] J. L. Marie, "Simple analytical formulation for micro-bubble drag reduction," Physico-Chemical Hydrodyn. , vol. 8, pp. 213-220, 1987.

[82] H. H. Legner, "A simple model for gas bubble drag reduction," Phys. Fluids , vol. 27, pp. 2788-2790, 1984.

[83] D. T. Jones, “Jones, D. T.," University of Wales, 1967.

[84] R. H. Thomas and K. Walters, "On the flow of an elastico-viscous liquid in a curved pipe of elliptic cross-section under a pressure-gradient," J. Fluid Mech. , vol. 21, no. 01, pp. 173-182, 1965.

[85] S. N. Shah, Ahmed Kamel, and Y. Zhou, "Drag reduction characteristics in straight and coiled tubing - An experimental study," J. Pet. Sci. Eng. , vol. 53, no. 3-4, pp. 179-188, 2006. 
[86] J. V. Kelkar and R. A. Mashelkar, "Drag reduction in dilute polymer solutions," J. Appl. Polym. Sci. , vol. 16 , no. 12 , pp. 3047-3062, 1972.

[87] B. K. Rao, "Turbulent heat transfer to power-law fluids in helical passages," Int. J. Heat Fluid Flow , vol. 15, no. 2, pp. 142-148, 1994.

[88] S. N. Shah and Y. Zhou, "An experimental study of drag reduction of polymer solutions in coiled tubing," SPE J. , no. August, pp. 7-8, 2003.

[89] F. Gallego and S. N. Shah, "Friction pressure correlations for turbulent flow of drag reducing polymer solutions in straight and coiled tubing," J. Pet. Sci. Eng., vol. 65, no. 3-4, pp. 147-161, 2009.

[90] A. M. Robertson and S. J. Muller, "Flow of Oldroyd-B fluids in curved pipes of circular and annular cross-section," Int. J. Non-Linear Mech. , vol. 1, no. 31, pp. 1-20, 1996.

[91] P. S. Virk and E. W. Merill, "In:Wells, C.S. (Ed.), The Onset of Dilute Polymer Solution Phenomena in Viscous Drag Reduction: Viscous Drag Reduction.," Plenum Press. New York, 1969.

[92] N. S. Berman, "Drag reduction by polymers," Annu. Rev. Fluid Mech. , vol. 10, pp. 47-64, 1978.

[93] K. Gasljevic, G. Aguilar, and E. F. Matthys, "On two distinct types of drag-reducing fluids, diameter scaling, and turbulent profiles," J. Nonnewton. Fluid Mech. , vol. 96, pp. 405-425, 2001.

[94] W. Interthal and H. Wilski, "Drag reduction experiments with very large pipes," Colloid Polym. Sci. , vol. 263, pp. 217-229, 1985.

[95] S. Nakagawa and T. J. Hanratty, "Particle image velocimetry measurements of flow over a wavy wall," Phys. Fluids, vol. 13, no. 11, pp. 3504-3507, 2001.

[96] H. R. Karami and D. Mowla, "Investigation of the effects of various parameters on pressure drop reduction in crude oil pipelines by drag reducing agents," J. Nonnewton. Fluid Mech. , vol. 177-178, pp. 37-45, Jun. 2012.

[97] H. L. Petrie, S. Deutsch, T. A. Brungart, and A. A. Fontaine, "Polymer drag reduction with surface roughness in flat-plate turbulent boundary layer flow," Exp. Fluids, vol. 35, pp. 8-23, 2003.

[98] R. H. Thomas and K. Walters, "On the flow of an elastico-viscous liquid in a curved pipe under a pressure gradient," J. Fluid Mech. , vol. 16, no. 2, pp. 228-242, 1963.

[99] M. Munekata, K. Matsuzaki, and H. Ohba, "A study on viscoelastic fluid flow in a square-section 90-degrees bend," J. Therm. Sci. , vol. 12, no. 4, pp. 337-343, 2003.

[100] W. M. Jones and O. H. Davies, "The flow of dilute aqueous solutions of macromolecules in various geometries : III . Bent pipes and porous materials," J. Phys. D Appl. Phys., vol. 9, pp. 753-770, 1976.

[101] T. Yokoyama and Y. Tomita, "Flow of dilute polymer solutions through curved bends," Bull. JSME , vol. 252 , no. 29 , pp. $1740-1745,1986$.

[102] M. M. E. Shatat, S. Yanase, T. Takami, and T. Hyakutake, "Pressure drop characteristics of water flow with micro-bubbles through helical pipes," Recent Adv. Fluid Mech. , pp. 32-37, 2009.

[103] H. Saffari, R. Moosavi, and G. E., "The effect of bubble in the pressure drop reduction in helical coil," Exp. Therm. Fluid Sci., vol. 51, pp. 251-256, 2013.

[104] W. Y. Soh and S. A. Berger, "Fully developed flow in a curved pipe of arbitrary curvature ratio," Intl. J. Num. Meth. Fluids, vol. 7, pp. 733-755, 1987.

[105] D. J. McConalogue and R. S. Srivastava, "Motion of a fluid in a curved tube," Proc. R. Soc. , vol. 307, pp. 37-53, 1968. 
[106] B. D. Coleman and W. Noll, "Foundations of linear viscoelasticity," Rev. Mod. Phys. , vol. 33, p. 239, 1961.

[107] B. A. Mujawar, "Flow of non-Newtonian fluids through helical coils," Ind. Eng. Chem. Process Des. Dev. , vol. 17, no. 1, pp. 22-27, 1978.

[108] C. F. Hsu and S. V. Patankar, "Analysis of laminar non-Newtonian flow and heat transfer in curved tubes," AIChE J. , vol. 28, pp. 610-616, 1982.

[109] G. I. Taylor, "The criterion for turbulence in curved pipes," Proc. Roy. Soc. London Ser. A, vol. 124, pp. 243-249, 1929.

[110] K. R. Sreenivasan and P. J. Strykowski, "Stabilization effects in flow through helically coiled pipes," Exp. Fluids, vol. 1, pp. 31-36, 1983.

[111] Y. Zhou and S. N. Shah, "New Friction Factor Correlations of Non-Newtonian Fluid Flow in Coiled Tubing," in SPE Asia Pacific Oil and Gas Conference and Exhibition, 2002, pp. 68-76.

[112] G. Astarita, G. Greco, and L. Nicodemo, "A phenomenological interpretation and correlation of drag reduction," AIChE J., vol. 15, no. 4, pp. 564-567, 1969.

[113] R. J. Gordon, "On the explanation and correlation of turbulent drag reduction in dilute macromolecular solutions," J. Appl. Polym. Sci., vol. 14, no. 8, pp. 2097-2105, 1970.

[114] R. A. Mashelkar and G. V. Devarajan, "Secondary flows of non-Newtonian fluids: Part III - Turbulent flow of visco-inelastic fluids in coiled tubes: A theoretical analysis and experimental verification," Trans. Instn. Chem. Eng., vol. 55, pp. 29-37, 1977.

[115] P. Mishra and S. N. Gupta, "Momentum transfer in curved pipes . 2 . Non-Newtonian fluids," Ind. Eng. Chem. Process Des. Dev. , vol. 18, no. 1, pp. 137-142, 1979.

[116] H. Inaba, "New challenge in advanced thermal energy transportation using functionally thermal fluids," Int. J. Therm. Sci. , vol. 139, pp. 991-1003, 2000.

[117] Y. Zhou and S. N. Shah, "Non-Newtonian fluid flow in coiled tubing: theoretical analysis and experimental verification," Soc. Pet. Eng. , vol. SPE 77708, pp. 3225-3237, 2002.

[118] R. C. McCann and C. G. Islas, "Frictional pressure loss during turbulent flow in coiled tubing," Soc. Pet. Eng. , pp. 1-6, 1996.

[119] T. Al-Wahaibi, N. Yusuf, Y. Al-Wahaibi, and A. Al-Ajmi, "Experimental study on the transition between stratified and non-stratified," Int. J. Multiph. Flow, vol. 38, pp. 126-135, 2012.

[120] T. Al-Wahaibi, Y. Al-Wahaibi, A. Al-Ajmi, R. Al-Hajri, N. Yusuf, and A. S. Olawale, "Experimental investigation on $\mathrm{fl}$ ow patterns and pressure gradient through two pipe diameters in horizontal oil - water flows," J. Pet. Sci. Eng. , vol. 122, pp. 266-273, 2014.

[121] L. C. Edomwonyi-Otu and P. Angeli, "Effects of polymer addition on pressure drop and interfacial waves in horizontal oil-water flows," Pet. Technol. Dev. J., vol. 2, no. July, pp. 41-48, 2014.

[122] T. Al-Wahaibi et al. , "Experimental investigation on the performance of drag reducing polymers through two pipe diameters in horizontal oil-water flows," Exp. Therm. Fluid Sci. , vol. 50, pp. 139-146, 2013.

[123] E. A. Mujawar and M. R. Rao, "Gas-non-Newtonian liquid two-phase flow in helical coils," Society , no. 1966, pp. 391-397, 1981.

[124] G. R. Rippel, C. M. Eidt Jr., and H. B. Jordon Jr., "Two-phase flow in a coiled tube," Ind. Eng. Chem. Proc. Des. Dev., vol. 5, pp. 32-39, 1966. 
[125] R. W. Lockhart and R. C. Martinelli, "Proposed correlation of data for isothermal two-phase, twocomponent flow in pipes.pdf," Chem. Eng. Prog. , vol. 45, no. 1, pp. 39-48, 1949.

[126] A. B. Biswas and S. K. Das, "Two-phase frictional pressure drop of gas-non-Newtonian liquid flow through helical coils in vertical orientation," Chem. Eng. Process. Process Intensif. , vol. 47, no. 5, pp. $816-826,2008$.

[127] A. K. Thandlam, T. K. Mandal, and S. K. Majumder, "Flow pattern transition, frictional pressure drop , and holdup of gas non-Newtonian fl uid fl ow in helical tube," Asia-Pac. J. Chem. Eng. , vol. 10, pp. 422-437, 2015.

[128] K. A. Thandlam, C. Das, and K. S. Majumder, "Flow pattern - based mass and heat transfer and frictional drag of gas - non - Newtonian liquid flow in helical coil : two - and three - phase systems," Heat Mass Transf., pp. 1-15, 2016.

[129] A. Benchabane and K. Bekkour, "Rheological properties of carboxymethyl cellulose ( CMC ) solutions," Colloid Polym Sci, vol. 286, no. September, pp. 1173-1180, 2008.

[130] L. P. Reiss and T. J. Hanratty, "Experimental Study of the Unsteady Nature of the Viscous Sublayer," A.I.Ch.E. J. , vol. 9, no. 2, pp. 154-160, 1963.

[131] A. H. Essadki, I. Nikov, and H. Delmas, "Local shear and skin friction on particles in three-phase fluidized beds," Chem. Eng. Sci., vol. 60, pp. 6034-6042, 2005.

[132] S. V. S. R. Krishna Bandaru and R. P. Chhabra, "Pressure drop for single and two-phase flow of non-Newtonian liquids in helical coils," Can. J. Chem. Eng., vol. 80, no. 2, pp. 315-321, 2002. 